\title{
Minimizing movements for forced anisotropic mean curvature flow of partitions with mobilities
}

\author{
Giovanni Bellettini \\ University of Siena, via Roma 56, 53100, Siena, Italy \\ International Centre for Theoretical Physics (ICTP), Strada Costiera \\ 11, 34151, Trieste, Italy (bellettini@diism.unisi.it)

\begin{abstract}
Antonin Chambolle
CMAP, Ecole Polytechnique, CNRS, 91128, Palaiseau Cedex, France (antonin.chambolle@cmap.polytechnique.fr)
\end{abstract}

\author{
Shokhrukh Kholmatov \\ University of Vienna, Oskar-Morgenstern-Platz 1, 1090, Vienna, Austria \\ (shokhrukh.kholmatov@univie.ac.at)
}

(MS Received 12 March 2020; accepted 12 July 2020)

\begin{abstract}
Under suitable assumptions on the family of anisotropies, we prove the existence of a weak global $1 /(n+1)$-Hölder continuous in time mean curvature flow with mobilities of a bounded anisotropic partition in any dimension using the method of minimizing movements. The result is extended to the case when suitable driving forces are present. We improve the Hölder exponent to $1 / 2$ in the case of partitions with the same anisotropy and the same mobility and provide a weak comparison result in this setting for a weak anisotropic mean curvature flow of a partition and an anisotropic mean curvature two-phase flow.
\end{abstract}

Keywords: mean curvature flow; partitions; minimizing movements; forcing; anisotropy; mobility

2010 Mathematics subject classification: 53C44, 49J45, 49J53

\section{Introduction}

Many processes in material sciences such as phase transformation, crystal growth, grain growth, stress-driven rearrangement instabilities, etc., can be modelled as geometric interface motions, in which surface tensions act as a principal driving force (see e.g., $[\mathbf{1 5}, \mathbf{4 0}, \mathbf{4 9}, \mathbf{5 1}]$ and references therein). A typical example of such a motion is anisotropic mean curvature flow: given a norm $\phi$ on $\mathbb{R}^{n}$ (called anisotropy), the equation for the anisotropic mean curvature flow of hypersurfaces parametrized as $\Gamma_{t}$ reads as

$$
\beta(\nu) V=-\operatorname{div}_{\Gamma_{t}}[\nabla \phi(\nu)] \text { on } \Gamma_{t},
$$

where $V$ denotes the normal velocity of $\Gamma_{t}$ in the direction of the unit outer normal $\nu$ of $\Gamma_{t}$ and $\beta$ is the mobility, a positive kinetic coefficient [29]. Anisotropic

(C) The Author(s), 2020. Published by Cambridge University Press. This is an Open Access article, distributed under the terms of the Creative Commons Attribution licence (http:// creativecommons.org/licenses/by/4.0/), which permits unrestricted re-use, distribution, and reproduction in any medium, provided the original work is properly cited. 
mean curvature flow is called crystalline provided the boundary of the Wulff shape $W_{\phi}:=\{\phi \leqslant 1\}$ lies on finitely many hyperplanes; in this quite interesting case, equation (1.1) must be properly interpreted, due to the nondifferentiability of $\phi$; see for instance $[\mathbf{2}, \mathbf{5}-\mathbf{7}, \mathbf{9}, \mathbf{1 7}, \mathbf{1 9}, \mathbf{2 0}, \mathbf{2 7}, \mathbf{2 8}, \mathbf{3 1}, \mathbf{3 2}, \mathbf{5 3}]$. Equation (1.1) (sometimes referred to as the two-phase evolution) can be generalized to the case of networks in the plane, and more generally to the case of partitions of space (sometimes called the multiphase case): here the evolving sets are intrinsically nonsmooth, since the presence of triple junctions (in the plane), or multiple lines, quadruple points (in space), etc., during the flow is unavoidable. It must be stressed that evolutions of partitions received recently a lot of attention from the mathematical community $[11,13,23,25,26,38,39,45,50,52]$ both as a natural generalization of the case of two phases, and because they model a variety of physical phenomena, such as grain growth and evolution of multicrystals $[\mathbf{8}, \mathbf{4 0}]$.

The presence of singularities at finite time is a common feature of mean curvature flow type motions, both in the two-phase case $[\mathbf{3 3 - 3 6 , 4 4}]$, and in the multiphase case (see for instance [45]). This phenomenon justifies to introduce and study some notion of weak solution, defined globally in time. This has been done in several different ways: just to quote a few, the Brakke varifold-solution [15], the viscosity solution (see [30] and references therein), the Ilmanen elliptic regularization [37], the level-set theoretic subsolution and the minimal barrier solution (see [12] and references therein), the Almgren-Taylor-Wang [1] and Luckhaus-Sturzenhecker [42] solutions, next included by De Giorgi into his notion of minimizing movement and generalized minimizing movement (GMM) $[\mathbf{2 1 , 2 2}]$; see also $[\mathbf{1 6}, \mathbf{2 4}, \mathbf{4 7}]$. Some of those solutions (e.g., the Brakke solution [15, 54], the GMM solution [14], the elliptic regularization [50]) can be adapted to treat the multiphase case at least in the Euclidean case, especially those that do not rely heavily on the comparison principle. Also, the existence of a distributional solution of mean curvature evolution of partitions on the torus using the time thresholding method introduced in [46] has been proved in $[41]$; see also $[39]$.

The aim of the present paper is to prove the existence of a GMM for anisotropic mean curvature flow of partitions with no restrictions on the space dimension, in the presence of a set of mobilities and forcing terms, and to point out some qualitative properties of this weak evolution, which are obtained via a comparison argument with a GMM of each single phase considered separately.

Let us recall the definition of GMM for partitions from [22] (see definition 2.6 for the notion of bounded partition).

Definition 1.1 Generalized minimizing movement for partitions. Let $\mathbb{P}_{b}(N+1)$ be the set of all bounded $(N+1)$-partitions of $\mathbb{R}^{n}$ (definition 2.6) endowed with the $L^{1}\left(\mathbb{R}^{n}\right)$-convergence, and let $\mathfrak{F}: \mathbb{P}_{b}(N+1) \times \mathbb{P}_{b}(N+1) \times[1,+\infty) \rightarrow$ $[-\infty,+\infty]$ be defined as

$$
\mathfrak{F}(\mathcal{A}, \mathcal{B}, \lambda)=\sum_{j=1}^{N+1} P_{\phi_{j}}\left(A_{j}\right)+\lambda \sum_{j=1}^{N+1} \int_{A_{j} \Delta B_{j}} d_{\psi_{j}}\left(x, \partial B_{j}\right) \mathrm{d} x+\sum_{j=1}^{N+1} \int_{A_{j}} H_{j} \mathrm{~d} x,
$$

where $\phi_{j}$ and $\psi_{j}$ are norms on $\mathbb{R}^{n}$, called anisotropies and mobilities, respectively, $H_{i} \in L_{\mathrm{loc}}^{1}\left(\mathbb{R}^{n}\right), \quad i=1, \ldots, N$, and $H_{N+1} \in L^{1}\left(\mathbb{R}^{n}\right)$ are driving forces, $P_{\phi_{j}}\left(A_{j}\right)$ 
is the $\phi_{j}$-anisotropic perimeter, $\mathcal{A}=\left(A_{1}, \ldots, A_{N+1}\right), \mathcal{B}=\left(B_{1}, \ldots, B_{N+1}\right)$ and $d_{\psi_{j}}(\cdot, E)$ is the $\psi_{j}$-distance function from $E \subseteq \mathbb{R}^{n}$. We say that a map $\mathcal{M}$ : $[0,+\infty) \rightarrow \mathbb{P}_{b}(N+1)$ is a $\mathrm{GMM}$ associated to $\mathfrak{F}$ starting from $\mathcal{G} \in \mathbb{P}_{b}(N+1)$, and we write $\mathcal{M} \in G M M(\mathfrak{F}, \mathcal{G})$, if there exist $\mathcal{L}:[1,+\infty) \times \mathbb{N}_{0} \rightarrow \mathbb{P}_{b}(N+1)$ and a diverging sequence $\left\{\lambda_{h}\right\}$ such that

$$
\lim _{h \rightarrow+\infty} \mathcal{L}\left(\lambda_{h},\left[\lambda_{h} t\right]\right)=\mathcal{M}(t) \quad \text { in } L^{1}\left(\mathbb{R}^{n}\right) \text { for any } t \geqslant 0,
$$

where the bounded partitions $\mathcal{L}(\lambda, k), \quad \lambda \geqslant 1, \quad k \in \mathbb{N}_{0}$, are defined inductively as $\mathcal{L}(\lambda, 0)=\mathcal{G}$ and

$$
\mathfrak{F}(\mathcal{L}(\lambda, k+1), \mathcal{L}(\lambda, k), \lambda)=\min _{\mathcal{A} \in \mathbb{P}_{b}(N+1)} \mathfrak{F}(\mathcal{A}, \mathcal{L}(\lambda, k), \lambda) \quad \forall k \geqslant 0 .
$$

Our first result (see theorems 4.1 and 4.2 for the precise statements) extends the existence results of $[\mathbf{1 4}]$ to the case with anisotropies, mobilities and external forces. We also improve the $1 /(n+1)$-Hölder regularity in time of GMM proven in $[\mathbf{1 4}]$ to $1 / 2$-Hölder continuity in the two-phase case, without any restriction on the anisotropies.

TheOREM 1.2. Suppose that the driving forces $\left\{H_{i}\right\}$ satisfy (4.4). Let $\mathcal{G} \in \mathbb{P}_{b}$ $(N+1)$. The following assertions hold:

(a) Let $N \geqslant 2$. If $\left\{\phi_{j}\right\}$ satisfy (3.1) and (4.3), then $G M M(\mathfrak{F}, \mathcal{G})$ is nonempty. Moreover, any $\mathcal{M}=\left(M_{1}, \ldots, M_{N+1}\right) \in G M M(\mathfrak{F}, \mathcal{G})$ is locally $1 /(n+1)$ Hölder continuous in time and for any $t \geqslant 0, \bigcup_{j=1}^{N} M_{j}(t)$ is contained in the bounded closed convex set related to $\mathcal{G}$ and $H_{j}$ (see (4.6)).

(b) Let $N=1$. Then, with no assumptions on the anisotropies $\phi_{1}, \phi_{2}$ and the mobilities $\psi_{1}, \psi_{2}, \quad G M M(\mathfrak{F}, \mathcal{G})$ is nonempty. Moreover, any $\mathcal{M} \in$ $\operatorname{GMM}(\mathfrak{F}, \mathcal{G})$ is locally 1/2-Hölder continuous in time.

To prove theorem 1.2 we establish uniform density estimates for minimizers of $\mathfrak{F}$ using the method of cutting out and filling in with balls, an argument of [42]. At this level the presence of mobilities does not create any new substantial problem. While in the two-phase case we do not need any assumption on the anisotropies, in the multiphase case assumption (3.1) is needed to get the lower volume density estimate for minimizers which is important in the proof of time-continuity of GMM.

In case of partitions with the same anisotropies and the same mobilities and without forcing, the Hölder exponent of GMM can be improved to $1 / 2$ (see theorem 5.1). Denoting by $\mathfrak{F}_{2}$ the restriction of $\mathfrak{F}$ to two-phase case without forcing (see (5.2)), this can be done using the comparison property (theorem 5.2) between the minimizers of $\mathfrak{F}$ and the minimizers of $\mathfrak{F}_{2}$. This comparison result also enables us to get a weak comparison flow of corresponding multiphase and two-phase flows (theorem 5.5):

Theorem 1.3. Assume that $\phi_{j}=\phi_{i}$ and $\psi_{j}=\psi_{i}$ for all $i, j=1, \ldots, N+1$, and $H_{i}=0$ for all $i=1, \ldots, N+1$. Then any $\mathcal{M} \in G M M(\mathfrak{F}, \mathcal{G})$ is locally $1 / 2$ Hölder continuous in time and $\bigcup_{j=1}^{N} M_{j}(t)$ is contained in the closed convex 
envelope of the union $\bigcup_{j=1}^{N} G_{j}$ of the bounded components of $\mathcal{G}$ for any $t \geqslant 0$. Moreover:

(a) for any $\mathcal{M} \in G M M(\mathfrak{F}, \mathcal{G})$ and for any $i \in\{1, \ldots, N+1\}$ there exists $L_{i} \in$ $G M M\left(\mathfrak{F}_{2}, G_{i}\right)$ such that

$$
L_{i}(t) \subseteq M_{i}(t), \quad t \geqslant 0
$$

(b) Let $C_{i}, \quad i \in\{1, \ldots, N\}$, and $C_{N+1}$ be any convex sets such that $C_{i} \subset G_{i}$ for any $i \in\{1, \ldots, N+1\}$ and let $L_{i} \in M M\left(\mathfrak{F}_{2}, C_{i}\right)$ be the unique minimizing movement starting from $C_{i}$. Then for any $\mathcal{M} \in G M M(\mathfrak{F}, \mathcal{G})$,

$$
L_{i}(t) \neq \emptyset \Longrightarrow M_{i}(t) \neq \emptyset \quad \text { for any } i \in\{1, \ldots, N\}
$$

and

$$
\mathbb{R}^{n} \backslash L_{N+1}(t)=\emptyset \Longrightarrow \mathbb{R}^{n} \backslash M_{N+1}(t)=\emptyset
$$

Note that the comparison principle (1.3) implies that any bounded partition will disappear in the long run; moreover, (1.2) allows to estimate the extinction time of the $i$-th bounded phase (Corollary 5.6).

Finally, let us mention that a natural problem remains open, namely the consistency of GMM with the classical solution, provided the latter exists, at least on a short time interval. Such a result has been proven by Almgren-Taylor-Wang in [1] in the two-phase case without mobility; the proof is based on various stability properties of the flow, and using comparison arguments. It has also been proven by Almgren-Taylor [2] in the two-phase crystalline case. However, consistency is not known in the case of networks in the plane (and a fortiori for partitions in space), even in the Euclidean case without mobilities and forcing.

The paper is organized as follows. In $\S 2$ we introduce the notation, some results from the theory of sets of finite perimeter, and the definition of a partition. In $\S 3$ we prove the density estimates for almost minimizers. The existence of generalized minimizing movements (theorem 1.2) is established in $\S 4$. In $\S 5$ we improve the Hölder regularity of GMM (theorem 1.3) and provide some weak comparison principles.

\section{Notation and preliminaries}

In this section, we introduce the notation and collect some important properties of sets of locally finite perimeter. The standard references for $B V$-functions and sets of finite perimeter are $[\mathbf{4}, \mathbf{4 3}]$.

We use $\mathbb{N}_{0}$ to denote the set of all nonnegative integers. The symbol $B_{r}(x)$ stands for the open ball in $\mathbb{R}^{n}$ centred at $x \in \mathbb{R}^{n}$ of radius $r>0$. The characteristic function of a Lebesgue measurable set $F$ is denoted by $\chi_{F}$ and its Lebesgue measure by $|F|$; we set also $\omega_{n}:=\left|B_{1}(0)\right|$. We denote by $E^{c}$ the complement of $E$ in $\mathbb{R}^{n}$. 
Given a norm $\psi$ in $\mathbb{R}^{n}$ and a nonempty set $E \subseteq \mathbb{R}^{n}, d_{\psi}(\cdot, E)$ stands for the $\psi$-distance from $E$, i.e.,

$$
d_{\psi}(x, E)=\inf \{\psi(x-y): y \in E\}
$$

and

$$
\widetilde{d}_{\psi}(x, \partial E)=d_{\psi}(x, E)-d_{\psi}\left(x, \mathbb{R}^{n} \backslash E\right)
$$

is the signed $\psi$-distance function from $\partial E$, negative inside $E$. When $\psi$ is Euclidean for simplicity we drop the dependence on $\psi$. We also write

$$
\operatorname{diam}_{\psi} E:=\sup \{\psi(x-y): x, y \in E\}
$$

to denote the $\psi$-diameter of $E$.

By $\mathbb{O}\left(\mathbb{R}^{n}\right)$ (resp. $\mathbb{O}_{b}\left(\mathbb{R}^{n}\right)$ ) we denote the collection of all open (resp. open and bounded) subsets of $\mathbb{R}^{n}$. The set of $L_{\text {loc }}^{1}\left(\mathbb{R}^{n}\right)$-functions having locally bounded total variation in $\mathbb{R}^{n}$ is denoted by $B V_{\text {loc }}\left(\mathbb{R}^{n}\right)$ and the elements of

$$
B V_{\mathrm{loc}}\left(\mathbb{R}^{n},\{0,1\}\right):=\left\{E \subseteq \mathbb{R}^{n}: \chi_{E} \in B V_{\mathrm{loc}}\left(\mathbb{R}^{n}\right)\right\}
$$

are called locally finite perimeter sets. Given a $E \in B V_{\text {loc }}\left(\mathbb{R}^{n},\{0,1\}\right)$ we denote by

(a) $P(E, \Omega):=\int_{\Omega}\left|D \chi_{E}\right|$ the perimeter of $E$ in $\Omega \in \mathbb{O}\left(\mathbb{R}^{n}\right)$;

(b) $\partial E$ the measure-theoretic boundary of $E$ :

$$
\partial E:=\left\{x \in \mathbb{R}^{n}: 0<\left|B_{\rho} \cap E\right|<\left|B_{\rho}\right| \quad \forall \rho>0\right\} ;
$$

(c) $\partial^{*} E$ the reduced boundary of $E$;

(d) $\nu_{E}$ the outer generalized unit normal to $\partial^{*} E$.

For simplicity, we set $P(E):=P\left(E, \mathbb{R}^{n}\right)$ provided $E \in B V\left(\mathbb{R}^{n} ;\{0,1\}\right)$. Further, given a Lebesgue measurable set $E \subseteq \mathbb{R}^{n}$ and $\alpha \in[0,1]$ we define

$$
E^{(\alpha)}:=\left\{x \in \mathbb{R}^{n}: \lim _{\rho \rightarrow 0^{+}} \frac{\left|B_{\rho}(x) \cap E\right|}{\left|B_{\rho}(x)\right|}=\alpha\right\} .
$$

Unless otherwise stated, we always suppose that any locally finite perimeter set $E$ we consider coincides with $E^{(1)}$ (so that by [43, equation (15.3)] $\partial E$ coincides with the topological boundary). We recall that $\overline{\partial^{*} E}=\partial E$ and $D \chi_{E}=\nu_{E} d \mathcal{H}^{n-1}\left\llcorner\partial^{*} E\right.$, where $\mathcal{H}^{n-1}$ is the $(n-1)$-dimensional Hausdorff measure in $\mathbb{R}^{n}$ and $L$ is the symbol of restriction.

REmark 2.1. Given $E \in B V_{\text {loc }}\left(\mathbb{R}^{n} ;\{0,1\}\right)$ the map $\Omega \in \mathbb{O}\left(\mathbb{R}^{n}\right) \mapsto P(E, \Omega)$ extends to a Borel measure in $\mathbb{R}^{n}$ so that $P(E, B)=\mathcal{H}^{n-1}\left(B \cap \partial^{*} E\right)$ for every Borel set $B \subseteq \mathbb{R}^{n}$. Moreover, by $\left[4\right.$, theorem 3.61] for every $E \in B V_{\text {loc }}\left(\mathbb{R}^{n} ;\{0,1\}\right)$

$$
\mathcal{H}^{n-1}\left(\mathbb{R}^{n} \backslash\left(E^{(0)} \cup E \cup \partial^{*} E\right)\right)=0 .
$$

In particular, $\mathcal{H}^{n-1}\left(E^{(1 / 2)} \backslash \partial^{*} E\right)=0$. 
Theorem 2.2. [43, theorem 16.3] If $E$ and $F$ are sets of locally finite perimeter, and we let

$$
\begin{gathered}
\left\{\nu_{E}=\nu_{F}\right\}=\left\{x \in \partial^{*} E \cap \partial^{*} F: \nu_{E}(x)=\nu_{F}(x)\right\}, \\
\left\{\nu_{E}=-\nu_{F}\right\}=\left\{x \in \partial^{*} E \cap \partial^{*} F: \nu_{E}(x)=-\nu_{F}(x)\right\},
\end{gathered}
$$

then $E \cap F, E \backslash F$ and $E \cup F$ are locally finite perimeter sets with

$$
\begin{aligned}
\partial^{*}(E \cap F) & \approx\left(F \cap \partial^{*} E\right) \cup\left(E \cap \partial^{*} F\right) \cup\left\{\nu_{E}=\nu_{F}\right\}, \\
\partial^{*}(E \backslash F) & \approx\left(F^{(0)} \cap \partial^{*} E\right) \cup\left(E \cap \partial^{*} F\right) \cup\left\{\nu_{E}=-\nu_{F}\right\}, \\
\partial^{*}(E \cup F) & \approx\left(F^{(0)} \cap \partial^{*} E\right) \cup\left(E^{(0)} \cap \partial^{*} F\right) \cup\left\{\nu_{E}=\nu_{F}\right\},
\end{aligned}
$$

where $A \approx B$ means $\mathcal{H}^{n-1}(A \Delta B)=0$.

The following generalizes the notion of the perimeter.

Definition 2.3 Anisotropic perimeter. Let $\phi: \mathbb{R}^{n} \rightarrow[0, \infty)$ be a norm in $\mathbb{R}^{n}$. Given $\Omega \in \mathbb{O}\left(\mathbb{R}^{n}\right)$ the $\phi$-perimeter of $E \in B V(\Omega ;\{0,1\})$ is

$$
P_{\phi}(E, \Omega):=\int_{\Omega \cap \partial^{*} E} \phi\left(\nu_{E}\right) \mathrm{d} \mathcal{H}^{n-1} .
$$

When $\Omega=\mathbb{R}^{n}$, we write $P_{\phi}(E):=P_{\phi}\left(E, \mathbb{R}^{n}\right)$, and when $\phi$ is Euclidean, we write $P$ in place of $P_{\phi}$.

It is well-known that $E \mapsto P_{\phi}(E ; \Omega)$ is $L_{\text {loc }}^{1}(\Omega)$-lower semicontinuous. Recall also that for every $E, F \in B V_{\text {loc }}\left(\mathbb{R}^{n} ;\{0,1\}\right)$ and $\Omega \in \mathbb{O}\left(\mathbb{R}^{n}\right)$

$$
P_{\phi}(E \cap F, \Omega)+P_{\phi}(E \cup F, \Omega) \leqslant P_{\phi}(E, \Omega)+P_{\phi}(F, \Omega) .
$$

\subsection{Anisotropic partitions}

We recall the notions of partition, almost-minimizer and bounded partition, see $[14]$.

Definition 2.4 Partition. Given an integer $N \geqslant 2$, an $N$-tuple $\mathcal{C}=$ $\left(C_{1}, \ldots, C_{N}\right)$ of subsets of $\mathbb{R}^{n}$ is called an $N$-partition of $\mathbb{R}^{n}$ (a partition, for short) if

(a) $C_{i} \in B V_{\text {loc }}\left(\mathbb{R}^{n} ;\{0,1\}\right)$ for every $i=1, \ldots, N$,

(b) $\sum_{i=1}^{N}\left|C_{i} \cap K\right|=|K|$ for each compact $K \subset \mathbb{R}^{n}$.

The collection of all $N$-partitions of $\mathbb{R}^{n}$ is denoted by $\mathbb{P}(N)$. Our assumptions $C_{i}=C_{i}^{(1)}$ imply $C_{i} \cap C_{j}=\emptyset$ for $i \neq j$.

The elements of $\mathbb{P}(N)$ are denoted by calligraphic letters $\mathcal{A}, \mathcal{B}, \mathcal{C}, \ldots$ and the components of $\mathcal{A} \in \mathbb{P}(N)$ by the corresponding roman letters $\left(A_{1}, \ldots, A_{N}\right)$. 
Let $\phi_{1}, \ldots, \phi_{N}$ be norms in $\mathbb{R}^{n}$ and set $\Phi:=\left\{\phi_{1}, \ldots, \phi_{N}\right\}$. The functional

$$
(\mathcal{A}, \Omega) \in \mathbb{P}(N) \times \mathbb{O}\left(\mathbb{R}^{n}\right) \mapsto \operatorname{Per}_{\Phi}(\mathcal{A}, \Omega):=\sum_{i=1}^{N} P_{\phi_{i}}\left(A_{i}, \Omega\right)
$$

is called the anisotropic perimeter, or $\Phi$-perimeter of the partition $\mathcal{A}$ in $\Omega$. For simplicity, we write $\operatorname{Per}_{\Phi}(\mathcal{A}):=\operatorname{Per}_{\Phi}\left(\mathcal{A}, \mathbb{R}^{n}\right)$. For shortness, we also set $\operatorname{Per}_{\Phi}=$ Per when all $\phi_{i}$ are Euclidean. Since $N$ is finite, there exist $0<c_{\Phi} \leqslant C_{\Phi}<+\infty$ such that

$$
c_{\Phi} \leqslant \phi_{i}(\nu) \leqslant C_{\Phi}
$$

for any $i=1, \ldots, N$ and $\nu \in \mathbb{S}^{n-1}$, therefore,

$$
c_{\Phi} \operatorname{Per}(\mathcal{A}, \Omega) \leqslant \operatorname{Per}_{\Phi}(\mathcal{A}, \Omega) \leqslant C_{\Phi} \operatorname{Per}(\mathcal{A}, \Omega) .
$$

In view of [14, proposition 3.3]

$$
\operatorname{Per}_{\Phi}(\mathcal{A}, \Omega)=\sum_{1 \leqslant i<j \leqslant N} \int_{\Omega \cap \partial^{*} A_{i} \cap \partial^{*} A_{j}}\left(\phi_{i}\left(\nu_{A_{i}}\right)+\phi_{j}\left(\nu_{A_{i}}\right)\right) \mathrm{d} \mathcal{H}^{n-1},
$$

i.e., on a generalized hypersurface $\Sigma_{i j}:=\Omega \cap \partial^{*} A_{i} \cap \partial^{*} A_{j}$ dividing the phase $i$ from the phase $j$ the perimeter contributes

$$
\int_{\Sigma_{i j}}\left(\phi_{i}\left(\nu_{\Sigma_{i j}}\right)+\phi_{j}\left(\nu_{\Sigma_{i j}}\right)\right) \mathrm{d} \mathcal{H}^{n-1}
$$

where $\nu_{\Sigma_{i j}}$ is the generalized unit normal to $\Sigma_{i j}$ pointing for instance from $A_{i}$ to $A_{j}$. We set

$$
\mathcal{A} \Delta \mathcal{B}:=\bigcup_{j=1}^{N} A_{j} \Delta B_{j} \quad \text { and } \quad|\mathcal{A} \Delta \mathcal{B}|:=\sum_{j=1}^{N}\left|A_{j} \Delta B_{j}\right|,
$$

where $\Delta$ is the symmetric difference of sets, i.e., $E \Delta F=(E \backslash F) \cup(F \backslash E)$. if

We say that the sequence $\left\{\mathcal{A}^{(k)}\right\} \subseteq \mathbb{P}(N)$ converges to $\mathcal{A} \in \mathbb{P}(N)$ in $L_{\text {loc }}^{1}\left(\mathbb{R}^{n}\right)$

$$
\left|\left(\mathcal{A}^{(k)} \Delta \mathcal{A}\right) \cap K\right|:=\sum_{j=1}^{N}\left|\left(A_{j}^{(k)} \Delta A_{j}\right) \cap K\right| \rightarrow 0 \quad \text { as } k \rightarrow+\infty
$$

for every compact set $K \subset \mathbb{R}^{n}$. Since $E \in B V_{\text {loc }}\left(\mathbb{R}^{n} ;\{0,1\}\right) \mapsto P_{\phi_{i}}(E, \Omega)$ is $L_{\text {loc }}^{1}\left(\mathbb{R}^{n}\right)$-lower semicontinuous for any $\Omega \in \mathbb{O}\left(\mathbb{R}^{n}\right)$, so is the map $\mathcal{A} \in \mathbb{P}(N) \mapsto$ $\operatorname{Per}_{\Phi}(\mathcal{A}, \Omega)$. From $(2.6)$ and $[\mathbf{1 4}$, theorem 3.2] we get

Proposition 2.5 Compactness. Let $\left\{\mathcal{A}^{(l)}\right\} \subset \mathbb{P}(N)$ be a sequence of partitions such that

$$
\sup _{l \geqslant 1} \operatorname{Per}_{\Phi}\left(\mathcal{A}^{(l)}, \Omega\right)<+\infty \quad \forall \Omega \in \mathbb{O}_{b}\left(\mathbb{R}^{n}\right) .
$$

Then there exist a partition $\mathcal{A} \in \mathbb{P}(N)$ and a subsequence $\left\{\mathcal{A}^{\left(l_{k}\right)}\right\}$ converging to $\mathcal{A}$ in $L_{\text {loc }}^{1}\left(\mathbb{R}^{n}\right)$ as $k \rightarrow+\infty$. 


\subsection{Bounded partitions}

Definition 2.6 Bounded partition. A partition $\mathcal{C}=\left(C_{1}, \ldots, C_{N+1}\right) \in \mathbb{P}(N+1)$ is called bounded, and we write $\mathcal{C} \in \mathbb{P}_{b}(N+1)$, if $C_{i}$ is bounded for each $i=$ $1, \ldots, N$.

Note that $\mathcal{A} \Delta \mathcal{B} \subset \subset \mathbb{R}^{n}$ for every $\mathcal{A}, \mathcal{B} \in \mathbb{P}_{b}(N+1)$, and therefore,

$$
|\mathcal{A} \Delta \mathcal{B}|=\sum_{j=1}^{N+1}\left|A_{j} \Delta B_{j}\right|
$$

is the $L^{1}\left(\mathbb{R}^{n}\right)$-distance in $\mathbb{P}_{b}(N+1)$.

Given $\mathcal{A} \in \mathbb{P}_{b}(N+1)$, we denote by $\operatorname{co}(\mathcal{A})$ the closed convex hull of $\bigcup_{i=1}^{N} A_{i}$. In view of (2.6) and [14, theorem 3.10] we have the following compactness result.

Proposition 2.7 Compactness. Let $\mathcal{A}^{(k)} \in \mathbb{P}_{b}(N+1), \quad k=1,2, \ldots$, and $\Omega \in$ $\mathbb{O}_{b}\left(\mathbb{R}^{n}\right)$ be such that

$$
\sup _{k \geqslant 1} \operatorname{Per}_{\Phi}\left(\mathcal{A}^{(k)}\right)<+\infty, \quad \operatorname{co}\left(\mathcal{A}^{(k)}\right) \subseteq \Omega \quad \forall k \geqslant 1
$$

Then there exist $\mathcal{A} \in \mathbb{P}_{b}(N+1)$ and a subsequence $\left\{\mathcal{A}^{\left(k_{l}\right)}\right\}$ converging to $\mathcal{A}$ in $L^{1}\left(\mathbb{R}^{n}\right)$ as $l \rightarrow+\infty$. Moreover, $\bigcup_{i=1}^{N} A_{j} \subseteq \bar{\Omega}$.

\section{Density estimates for almost minimizers}

In this section we prove density estimates for almost minimizers (see theorem 3.2). In the two-phase case without mobility, density estimates have been proven in $[\mathbf{1}, \mathbf{4 2}]$ (see also the proof of theorem 4.2 for the case with mobility and forcing) and in the isotropic $N$-phase case is proven in [14]. The proof of theorem 3.2 is similar to [14, theorem 3.6], however, some (technical) difficulties arise when two anisotropies differ too much and this is why we need assumption (3.5) for proving the lower-density estimates.

Definition 3.1 Almost-minimizers. Given $\Phi=\left\{\phi_{1}, \ldots, \phi_{N}\right\}, \Lambda_{1}, \Lambda_{2} \geqslant 0, \alpha_{1}, \alpha_{2}>$ $(n-1) / n$ and $r_{0} \in(0,+\infty]$, we say that a partition $\mathcal{A} \in \mathbb{P}(N)$ is a $\left(\Phi, \Lambda_{1}, \Lambda_{2}, r_{0}, \alpha_{1}, \alpha_{2}\right)$-minimizer in $\mathbb{R}^{n}$ of $\operatorname{Per}_{\Phi}\left(\right.$ a $\left(\Lambda_{1}, \Lambda_{2}, r_{0}, \alpha_{1}, \alpha_{2}\right)$-minimizer, or also an almost-minimizer for short) if

$$
\operatorname{Per}_{\Phi}\left(\mathcal{A}, B_{r}\right) \leqslant \operatorname{Per}_{\Phi}\left(\mathcal{B}, B_{r}\right)+\Lambda_{1}|\mathcal{A} \Delta \mathcal{B}|^{\alpha_{1}}+\Lambda_{2}|\mathcal{A} \Delta \mathcal{B}|^{\alpha_{2}}
$$

whenever $\mathcal{B} \in \mathbb{P}(N), \quad B_{r} \subset \mathbb{R}^{n}$ is a ball of radius $r \in\left(0, r_{0}\right)$ and $\mathcal{A} \Delta \mathcal{B} \subset \subset B_{r}$. 
Define

$$
\begin{aligned}
\kappa_{N} & :=\min _{1 \leqslant i<j \leqslant N}\left\|\phi_{i}-\phi_{j}\right\|_{L^{\infty}\left(\mathbb{S}^{n-1}\right)}, \\
\beta_{1} & :=\left(\frac{c_{\Phi} n \omega_{n}^{1-\alpha_{1}}}{2^{1+\alpha_{1}} \Lambda_{1}}\right)^{1 /\left(n \alpha_{1}-n+1\right)}, \\
\beta_{2} & :=\left(\frac{c_{\Phi} n \omega_{n}^{1-\alpha_{2}}}{2^{1+\alpha_{2}} \Lambda_{2}}\right)^{1 /\left(n \alpha_{2}-n+1\right)}, \\
\gamma_{N} & :=\frac{c_{\Phi}-(N-1) \kappa_{N} / 2}{2 c_{\Phi}+2(N-1) C_{\Phi}-(N-1) \kappa_{N}} .
\end{aligned}
$$

Theorem 3.2 Density estimates for almost minimizers. Assume that the entries of $\Phi$ satisfy (2.5). Let $\mathcal{A} \in \mathbb{P}(N)$ be a $\left(\Lambda_{1}, \Lambda_{2}, r_{0}, \alpha_{1}, \alpha_{2}\right)$-minimizer and $i \in\{1, \ldots, N\}$. Then either $A_{i}=\emptyset$ or for any $x \in \partial A_{i}$ and $r \in\left(0, \widehat{r}_{0}\right]$

$$
\frac{\left|A_{i} \cap B_{r}(x)\right|}{\left|B_{r}(x)\right|} \leqslant 1-\left(\frac{c_{\Phi}}{2\left(c_{\Phi}+C_{\Phi}\right)}\right)^{n}
$$

and

$$
\frac{P\left(A_{i}, B_{r}(x)\right)}{r^{n-1}} \leqslant\left(\frac{C_{\Phi}}{c_{\Phi}}+\frac{1}{2}\right) n \omega_{n}
$$

where

$$
\widehat{r}_{0}:=\min \left\{r_{0}, \beta_{1}, \beta_{2}\right\}
$$

Moreover, if

$$
\kappa_{N}<\frac{2 c_{\Phi}}{N-1}
$$

then for any $r \in\left(0, \widetilde{r}_{0}\right]$

$$
\gamma_{N}^{n} \leqslant \frac{\left|A_{i} \cap B_{r}(x)\right|}{\left|B_{r}(x)\right|}
$$

and

$$
c \leqslant \frac{P\left(A_{i}, B_{r}(x)\right)}{r^{n-1}}
$$

where

$$
\widetilde{r}_{0}:=\min \left\{r_{0},\left(\frac{c_{\Phi}}{N-1}-\frac{\kappa_{N}}{2}\right)^{1 /\left(n \alpha_{1}-n+1\right)} \beta_{1},\left(\frac{c_{\Phi}}{N-1}-\frac{\kappa_{N}}{2}\right)^{1 /\left(n \alpha_{2}-n+1\right)} \beta_{2}\right\}
$$

and

$$
c:=c\left(n, N, c_{\Phi}, C_{\Phi}, \kappa_{N}\right):=\frac{n \omega_{n}\left(2^{1 / n}-1\right)}{2^{1+1 / n}} \gamma_{N}^{n-1}
$$

Proof. Without loss of generality, we assume $i=1$ and $A_{i} \neq \emptyset$. Since $\overline{\partial^{*} A_{1}}=$ $\partial A_{1}$, it is sufficient to show $(3.2),(3.3),(3.6),(3.7)$ when $x \in \partial^{*} A_{1}$. For shortness, we write $B_{r}:=B_{r}(x)$. 
We start by proving (3.2) and (3.3). Let us show

$$
\begin{aligned}
c_{\Phi} P\left(A_{1}^{(0)}, B_{r}\right) \leqslant & C_{\Phi} \mathcal{H}^{n-1}\left(A_{1}^{(0)} \cap \partial B_{r}\right) \\
& +2^{\alpha_{1}-1} \Lambda_{1}\left|A_{1}^{(0)} \cap B_{r}\right|^{\alpha_{1}}+2^{\alpha_{2}-1} \Lambda_{2}\left|A_{1}^{(0)} \cap B_{r}\right|^{\alpha_{2}}
\end{aligned}
$$

for all $r \in\left(0, \widehat{r}_{0}\right)$ such that

$$
\sum_{j=1}^{N} \mathcal{H}^{n-1}\left(\partial B_{r} \cap \partial^{*} A_{j}\right)=0
$$

Indeed, setting

$$
\mathcal{B}:=\left(A_{1} \cup B_{r}, A_{2} \backslash B_{r}, \ldots, A_{N} \backslash B_{r}\right),
$$

we have $\mathcal{A} \Delta \mathcal{B} \subset \subset B_{s}$ for every $s \in\left(r, \widehat{r}_{0}\right)$ and thus, by almost minimality, the definition $(2.7)$ of $|\mathcal{A} \Delta \mathcal{B}|$ and the essential disjointness of $A_{j}$,

$$
\begin{aligned}
0 \leqslant & \operatorname{Per}_{\Phi}\left(\mathcal{B}, B_{s}\right)-\operatorname{Per}_{\Phi}\left(\mathcal{A}, B_{s}\right)+\Lambda_{1}|\mathcal{A} \Delta \mathcal{B}|^{\alpha_{1}}+\Lambda_{2}|\mathcal{A} \Delta \mathcal{B}|^{\alpha_{2}} \\
= & P_{\phi_{1}}\left(A_{1} \cup B_{r}, B_{s}\right)-P_{\phi_{1}}\left(A_{1}, B_{s}\right)+\sum_{j=2}^{N}\left(P_{\phi_{j}}\left(A_{j} \backslash B_{r}, B_{s}\right)-P_{\phi_{j}}\left(A_{j}, B_{s}\right)\right) \\
& +2^{\alpha_{1}} \Lambda_{1}\left|B_{r} \cap A_{1}^{(0)}\right|^{\alpha_{1}}+2^{\alpha_{2}} \Lambda_{2}\left|B_{r} \cap A_{1}^{(0)}\right|^{\alpha_{2}}
\end{aligned}
$$

since $B_{r} \backslash A_{1}=B_{r} \cap A_{1}^{(0)}$ up to a $\mathcal{L}^{n}$-negligible set and $|\mathcal{A} \Delta \mathcal{B}|=2\left|B_{r} \backslash A_{1}\right|=$ $2\left|B_{r} \cap A_{1}^{(0)}\right|$. By $(2.3)$ and (3.10),

$$
P_{\phi_{1}}\left(A_{1} \cup B_{r}, B_{s}\right)=P_{\phi_{1}}\left(A_{1}, B_{s} \backslash \overline{B_{r}}\right)+\int_{A_{1}^{(0)} \cap \partial B_{r}} \phi_{1}\left(\nu_{B_{r}}\right) \mathrm{d} \mathcal{H}^{n-1},
$$

and for any $j=2, \ldots, N$,

$$
P_{\phi_{j}}\left(A_{j} \backslash B_{r}, B_{s}\right)=P_{\phi_{j}}\left(A_{j}, B_{s} \backslash \overline{B_{r}}\right)+\int_{A_{j} \cap \partial B_{r}} \phi_{j}\left(\nu_{B_{r}}\right) \mathrm{d} \mathcal{H}^{n-1} .
$$

Thus by (3.11)

$$
\begin{aligned}
\sum_{j=1}^{N} P_{\phi_{j}}\left(A_{j}, B_{s}\right) \leqslant & \sum_{j=1}^{N} P_{\phi_{j}}\left(A_{j}, B_{s} \backslash \overline{B_{r}}\right) \\
& +\int_{A_{1}^{(0)} \cap \partial B_{r}} \phi_{1}\left(\nu_{B_{r}}\right) \mathrm{d} \mathcal{H}^{n-1}+\sum_{j=2}^{N} \int_{A_{j} \cap \partial B_{r}} \phi_{j}\left(\nu_{B_{r}}\right) \mathrm{d} \mathcal{H}^{n-1} \\
& +2^{\alpha_{1}} \Lambda_{1}\left|B_{r} \cap A_{1}^{(0)}\right|^{\alpha_{1}}+2^{\alpha_{2}} \Lambda_{2}\left|B_{r} \cap A_{1}^{(0)}\right|^{\alpha_{2}}
\end{aligned}
$$


By (2.5), the essential disjointness of $A_{j}$ and (3.10) we have

$$
\begin{aligned}
& \int_{A_{1}^{(0)} \cap \partial B_{r}} \phi_{1}\left(\nu_{B_{r}}\right) \mathrm{d} \mathcal{H}^{n-1}+\sum_{j=2}^{N} \int_{A_{j} \cap \partial B_{r}} \phi_{j}\left(\nu_{B_{r}}\right) \mathrm{d} \mathcal{H}^{n-1} \\
& \leqslant C_{\Phi} \mathcal{H}^{n-1}\left(A_{1}^{(0)} \cap \partial B_{r}\right)+C_{\Phi} \sum_{j=2}^{N} \mathcal{H}^{n-1}\left(A_{j} \cap \partial B_{r}\right)=2 C_{\Phi} \mathcal{H}^{n-1}\left(A_{1}^{(0)} \cap \partial B_{r}\right),
\end{aligned}
$$

thus, (3.13) and (3.10) imply

$$
\sum_{j=1}^{N} P_{\phi_{j}}\left(A_{j}, B_{r}\right) \leqslant 2 C_{\Phi} \mathcal{H}^{n-1}\left(A_{1}^{(0)} \cap \partial B_{r}\right)+2^{\alpha_{1}} \Lambda_{1}\left|B_{r} \backslash A_{1}\right|^{\alpha_{1}}+2^{\alpha_{2}} \Lambda_{2}\left|B_{r} \backslash A_{1}\right|^{\alpha_{2}} .
$$

By (2.5), (2.4) and the essential disjointness of $A_{j}$,

$$
\sum_{j=2}^{N} P_{\phi_{j}}\left(A_{j}, B_{r}\right) \geqslant c_{\Phi} \sum_{j=2}^{N} P\left(A_{j}, B_{r}\right) \geqslant c_{\Phi} P\left(\bigcup_{j=2}^{N} A_{j}, B_{r}\right)=c_{\Phi} P\left(A_{1}^{(0)}, B_{r}\right),
$$

and thus $\sum_{j=1}^{N} P_{\phi_{j}}\left(A_{j}, B_{r}\right) \geqslant 2 c_{\Phi} P\left(A_{1}^{(0)}, B_{r}\right)$ so that (3.9) follows from (3.4).

To prove (3.2) we add $c_{\Phi} \mathcal{H}^{n-1}\left(A_{1}^{(0)} \cap \partial B_{r}\right)$ to both sides of (3.9) and using $\mathcal{H}^{n-1}\left(\partial B_{r} \cap \partial^{*} A_{1}\right)=0$ we get

$$
\begin{aligned}
c_{\Phi} P\left(A_{1}^{(0)} \cap B_{r}\right) \leqslant & \left(c_{\Phi}+C_{\Phi}\right) \mathcal{H}^{n-1}\left(A_{1}^{(0)} \cap \partial B_{r}\right)+2^{\alpha_{1}-1} \Lambda_{1}\left|A_{1}^{(0)} \cap B_{r}\right|^{\alpha_{1}} \\
& +2^{\alpha_{2}-1} \Lambda_{2}\left|A_{1}^{(0)} \cap B_{r}\right|^{\alpha_{2}},
\end{aligned}
$$

hence by the isoperimetric inequality

$$
\begin{aligned}
c_{\Phi} n \omega_{n}^{1 / n}\left|A_{1}^{(0)} \cap B_{r}\right|^{(n-1 / n} \leqslant & \left(c_{\Phi}+C_{\Phi}\right) \mathcal{H}^{n-1}\left(A_{1}^{(0)} \cap \partial B_{r}\right) \\
& +2^{\alpha_{1}-1} \Lambda_{1}\left|A_{1}^{(0)} \cap B_{r}\right|^{\alpha_{1}}+2^{\alpha_{2}-1} \Lambda_{2}\left|A_{1}^{(0)} \cap B_{r}\right|^{\alpha_{2}} .
\end{aligned}
$$

By the choice of $\widehat{r}_{0}$ in (3.4) we have, for $l=1,2$,

$$
2^{\alpha_{l}-1} \Lambda_{l}\left|A_{1}^{(0)} \cap B_{r}\right|^{\alpha_{l}-((n-1) / n)} \leqslant 2^{\alpha_{l}-1} \Lambda_{l} \omega_{n}^{\alpha_{l}-((n-1) / n)} \widehat{r}_{0}^{n \alpha_{l}-n+1} \leqslant \frac{c_{\Phi} n \omega_{n}^{1 / n}}{4} .
$$

Inserting (3.15) in (3.14) we obtain

$$
\frac{c_{\Phi}}{2\left(c_{\Phi}+C_{\Phi}\right)} n \omega_{n}^{1 / n}\left|A_{1}^{(0)} \cap B_{r}\right|^{(n-1 / n} \leqslant \mathcal{H}^{n-1}\left(A_{1}^{(0)} \cap \partial B_{r}\right)
$$

and whence, repeating for instance the arguments of the proof of [14, equation (3.19)], we obtain

$$
\left|A_{1}^{(0)} \cap B_{r}\right| \geqslant\left(\frac{c_{\Phi}}{2\left(c_{\Phi}+C_{\Phi}\right)}\right)^{n} \omega_{n} r^{n}
$$


i.e.,

$$
\frac{\left|A_{1} \cap B_{r}\right|}{\left|B_{r}\right|} \leqslant 1-\left(\frac{c_{\Phi}}{2\left(c_{\Phi}+C_{\Phi}\right)}\right)^{n} .
$$

From (3.9) and the definition of $\widehat{r}_{0}$ for all $r \in\left(0, \widehat{r}_{0}\right]$ we get

$$
\begin{aligned}
P\left(A_{1}, B_{r}\right) & \leqslant \frac{C_{\Phi}}{c_{\Phi}} \mathcal{H}^{n-1}\left(\partial B_{r}\right)+\frac{2^{\alpha_{1}-1} \Lambda_{1}}{c_{\Phi}}\left|B_{r}\right|^{\alpha_{1}}+\frac{2^{\alpha_{2}-1} \Lambda_{2}}{c_{\Phi}}\left|B_{r}\right|^{\alpha_{2}} \\
& \leqslant\left(\frac{C_{\Phi}}{c_{\Phi}}+\frac{1}{2}\right) n \omega_{n} r^{n-1} .
\end{aligned}
$$

Now we prove (3.6) and (3.7). Note that assumption (3.5) implies $\widetilde{r}_{0}, \gamma_{N}>0$. Let us show

$$
\begin{aligned}
\left(\frac{c_{\Phi}}{N-1}-\frac{\kappa_{N}}{2}\right) P\left(A_{1}, B_{r}\right) \leqslant & C_{\Phi} \mathcal{H}^{n-1}\left(A_{1} \cap \partial B_{r}\right) \\
& +2^{\alpha_{1}-1} \Lambda_{1}\left|A_{1} \cap B_{r}\right|^{\alpha_{1}}+2^{\alpha_{2}-1} \Lambda_{2}\left|A_{1} \cap B_{r}\right|^{\alpha_{2}}
\end{aligned}
$$

for all $r \in\left(0, \widetilde{r}_{0}\right)$ such that

$$
\sum_{j=1}^{N} \mathcal{H}^{n-1}\left(\partial^{*} A_{j} \cap \partial B_{r}\right)=0 .
$$

Set

$$
I_{1}:=\left\{j \in\{2, \ldots, N\}: \mathcal{H}^{n-1}\left(B_{\widetilde{r}_{0}} \cap \partial^{*} A_{1} \cap \partial^{*} A_{j}\right)>0\right\} .
$$

Since $x \in \partial A_{1}, \quad I_{1} \neq \emptyset$. Fix $r \in\left(0, \widetilde{r}_{0}\right)$; for every $j \in I_{1}$ consider the competitor

$$
\mathcal{B}^{(j)}:=\left(A_{1} \backslash B_{r}, A_{2}, \ldots, A_{j-1}, A_{j} \cup\left(A_{1} \cap B_{r}\right), A_{j+1}, \ldots, A_{N}\right) .
$$

Since $\mathcal{B}^{(j)} \Delta \mathcal{A} \subset \subset B_{s}$ for every $s \in\left(r, \widetilde{r}_{0}\right)$, by the almost minimality of $\mathcal{A}$ (recall that $\left.\widetilde{r}_{0} \leqslant r_{0}\right)$ and the equality $\left|\mathcal{A} \Delta \mathcal{B}^{(j)}\right|=2\left|A_{1} \cap B_{r}\right|$ one has

$$
\begin{aligned}
P_{\phi_{1}}\left(A_{1}, B_{s}\right)+P_{\phi_{j}}\left(A_{j}, B_{s}\right) \leqslant & P_{\phi_{1}}\left(A_{1} \backslash B_{r}, B_{s}\right)+P_{\phi_{j}}\left(A_{j} \cup\left(A_{1} \cap B_{r}\right), B_{s}\right) \\
& +2^{\alpha_{1}} \Lambda_{1}\left|A_{1} \cap B_{r}\right|^{\alpha_{1}}+2^{\alpha_{2}} \Lambda_{2}\left|A_{1} \cap B_{r}\right|^{\alpha_{2}} .
\end{aligned}
$$

Using the equality

$$
\begin{aligned}
P_{\phi_{j}}\left(A_{j} \cup\left(A_{1} \cap B_{r}\right), B_{s}\right)= & P_{\phi_{j}}\left(A_{j}, B_{s}\right)+P_{\phi_{j}}\left(A_{1}, B_{r}\right)+\int_{A_{1} \cap \partial B_{r}} \phi_{j}\left(\nu_{B_{r}}\right) \mathrm{d} \mathcal{H}^{n-1} \\
& -\int_{B_{r} \cap \partial^{*} A_{1} \cap \partial^{*} A_{j}}\left(\phi_{j}\left(\nu_{A_{1}}\right)+\phi_{j}\left(\nu_{A_{j}}\right)\right) \mathrm{d} \mathcal{H}^{n-1},
\end{aligned}
$$


the analogue of (3.12) with $j=1$ and also (3.17) in (3.18) we establish

$$
\begin{aligned}
P_{\phi_{1}}\left(A_{1}, B_{s}\right)+P_{\phi_{j}}\left(A_{j}, B_{s}\right) \leqslant & P_{\phi_{j}}\left(A_{j}, B_{s}\right)+P_{\phi_{j}}\left(A_{1}, B_{r}\right)+\int_{A_{1} \cap \partial B_{r}} \phi_{j}\left(\nu_{B_{r}}\right) \mathrm{d} \mathcal{H}^{n-1} \\
& -\int_{B_{r} \cap \partial^{*} A_{1} \cap \partial^{*} A_{j}}\left(\phi_{j}\left(\nu_{A_{1}}\right)+\phi_{j}\left(\nu_{A_{j}}\right)\right) \mathrm{d} \mathcal{H}^{n-1} \\
& +P_{\phi_{1}}\left(A_{1}, B_{s} \backslash B_{r}\right)+\int_{A_{1} \cap \partial B_{r}} \phi_{1}\left(\nu_{B_{r}}\right) \mathrm{d} \mathcal{H}^{n-1} \\
& +2^{\alpha_{1}} \Lambda_{1}\left|A_{1} \cap B_{r}\right|^{\alpha_{1}}+2^{\alpha_{2}} \Lambda_{2}\left|A_{1} \cap B_{r}\right|^{\alpha_{2}} .
\end{aligned}
$$

Hence using $\phi_{j}\left(\nu_{A_{1}}\right)=\phi_{j}\left(\nu_{A_{j}}\right)$,

$$
\begin{aligned}
& 2 \int_{B_{r} \cap \partial^{*} A_{1} \cap \partial^{*} A_{j}} \phi_{j}\left(\nu_{A_{1}}\right) \mathrm{d} \mathcal{H}^{n-1} \leqslant P_{\phi_{j}}\left(A_{1}, B_{r}\right)-P_{\phi_{1}}\left(A_{1}, B_{r}\right) \\
& +\int_{A_{1} \cap \partial B_{r}}\left(\phi_{1}\left(\nu_{B_{r}}\right)+\phi_{j}\left(\nu_{B_{r}}\right)\right) \mathrm{d} \mathcal{H}^{n-1}+2^{\alpha_{1}} \Lambda_{1}\left|A_{1} \cap B_{r}\right|^{\alpha_{1}}+2^{\alpha_{2}} \Lambda_{2}\left|A_{1} \cap B_{r}\right|^{\alpha_{2}} .
\end{aligned}
$$

Summing these inequalities in $j \in I_{1}$ and using (2.5) we get

$$
\begin{aligned}
2 c_{\Phi} \sum_{j=2}^{N} \mathcal{H}^{n-1}\left(B_{r} \cap \partial^{*} A_{1} \cap \partial^{*} A_{j}\right) \leqslant & \sum_{j \in I_{1}}\left(P_{\phi_{j}}\left(A_{1}, B_{r}\right)-P_{\phi_{1}}\left(A_{1}, B_{r}\right)\right) \\
& +\sum_{i \in I_{1}} \int_{A_{1} \cap \partial B_{r}}\left(\phi_{1}\left(\nu_{B_{r}}\right)+\phi_{j}\left(\nu_{B_{r}}\right)\right) \mathrm{d} \mathcal{H}^{n-1} \\
& +\left|I_{1}\right|\left(2^{\alpha_{1}} \Lambda_{1}\left|A_{1} \cap B_{r}\right|^{\alpha_{1}}+2^{\alpha_{2}} \Lambda_{2}\left|A_{1} \cap B_{r}\right|^{\alpha_{2}}\right),
\end{aligned}
$$

where $\left|I_{1}\right|$ is the number of elements of $I_{1}$. By the definition of $I_{1}$,

$$
\sum_{j \in I_{1}} \mathcal{H}^{n-1}\left(B_{r} \cap \partial^{*} A_{1} \cap \partial^{*} A_{j}\right)=P\left(A_{1}, B_{r}\right),
$$

by the definition of $\kappa_{N}$ in (3.1)

$$
\sum_{j \in I_{1}}\left(P_{\phi_{j}}\left(A_{1}, B_{r}\right)-P_{\phi_{1}}\left(A_{1}, B_{r}\right)\right) \leqslant \kappa_{N}\left|I_{1}\right| P\left(A_{1}, B_{r}\right),
$$

and by (2.5)

$$
\sum_{i \in I_{1}} \int_{A_{1} \cap \partial B_{r}}\left(\phi_{1}\left(\nu_{B_{r}}\right)+\phi_{j}\left(\nu_{B_{r}}\right)\right) \mathrm{d} \mathcal{H}^{n-1} \leqslant 2 C_{\Phi}\left|I_{1}\right| \mathcal{H}^{n-1}\left(A_{1} \cap \partial B_{r}\right) .
$$

Therefore, from (3.19) we obtain

$$
\begin{aligned}
\left(\frac{c_{\Phi}}{\left|I_{1}\right|}-\frac{\kappa_{N}}{2}\right) P\left(A_{1}, B_{r}\right) \leqslant & C_{\Phi} \mathcal{H}^{n-1}\left(A_{1} \cap \partial B_{r}\right)+2^{\alpha_{1}-1} \Lambda_{1}\left|A_{1} \cap B_{r}\right|^{\alpha_{1}} \\
& +2^{\alpha_{2}-1} \Lambda_{2}\left|A_{1} \cap B_{r}\right|^{\alpha_{2}} .
\end{aligned}
$$

Since $\left|I_{1}\right| \leqslant N-1$, inequality (3.16) follows. 
To prove (3.6) we add $\left(c_{\Phi} / N-1-\kappa_{N} / 2\right) \mathcal{H}^{n-1}\left(A_{1} \cap \partial B_{r}\right)$ to both sides of (3.16) and get

$$
\begin{aligned}
\left(\frac{c_{\Phi}}{N-1}-\frac{\kappa_{N}}{2}\right) P\left(A_{1} \cap B_{r}\right) \leqslant & \left(\frac{c_{\Phi}}{N-1}-\frac{\kappa_{N}}{2}+C_{\Phi}\right) \mathcal{H}^{n-1}\left(A_{1} \cap \partial B_{r}\right) \\
& +2^{\alpha_{1}-1} \Lambda_{1}\left|A_{1} \cap B_{r}\right|^{\alpha_{1}}+2^{\alpha_{2}-1} \Lambda_{2}\left|A_{1} \cap B_{r}\right|^{\alpha_{2}}
\end{aligned}
$$

By the definition (3.8) of $\widetilde{r}_{0}$ we have $r \leqslant \widetilde{r}_{0} \leqslant\left(c_{\Phi} / N-1-\kappa_{N} / 2\right)^{1 / n \alpha_{l}-n+1} \beta_{l}$ for $l=1,2$ and therefore

$$
\begin{aligned}
2^{\alpha_{l}-1} \Lambda_{l}\left|A_{1} \cap B_{r}\right|^{\alpha_{l}-n-1 / n} & \leqslant 2^{\alpha_{l}-1} \Lambda_{l}\left|B_{\widetilde{r}_{0}}\right|^{\alpha_{l}-n-1 / n} \\
& =\left(\frac{c_{\Phi}}{N-1}-\frac{\kappa_{N}}{2}\right) \frac{n \omega_{n}^{1 / n}}{4}, \quad l=1,2,
\end{aligned}
$$

and thus, by (3.20) and the isoperimetric inequality,

$$
\frac{c_{\Phi}-(N-1) \kappa_{N} / 2}{2 c_{\Phi}+2(N-1) C_{\Phi}-(N-1) \kappa_{N}} n \omega_{n}^{1 / n}\left|A_{1} \cap B_{r}\right|^{n-1 / n} \leqslant \mathcal{H}^{n-1}\left(A_{1} \cap \partial B_{r}\right) .
$$

Now integrating we get

$$
\gamma_{N}^{n} \omega_{n} r^{n} \leqslant\left|A_{1} \cap B_{r}\right|
$$

and (3.6) follows. Finally, since

$$
\frac{c_{\Phi}}{2 c_{\Phi}+2 C_{\Phi}}>\gamma_{N}
$$

from (3.2), (3.6) and the relative isoperimetric inequality we deduce (3.7).

The following volume-distance comparison appeared in a similar form also in $[\mathbf{1}, \mathbf{1 4}, \mathbf{4 2}]$ and will be used in the proof of the existence of GMM.

Proposition 3.3. Given $\theta, r_{0}>0$, let $A \in B V\left(\mathbb{R}^{n} ;\{0,1\}\right)$ be such that

$$
\theta r^{n-1} \leqslant P\left(A, B_{r}(x)\right), \quad r \in\left(0, r_{0}\right]
$$

whenever $x \in \partial A$. Then for any $\ell>0$ and $B \in B V\left(\mathbb{R}^{n} ;\{0,1\}\right)$ one has

$$
|B \Delta A| \leqslant \frac{5^{n} \omega_{n}}{\theta} \max \left\{1,\left(\frac{\ell}{r_{0}}\right)^{n-1}\right\} P(A) \ell+\frac{1}{\ell} \int_{A \Delta B} d(x, \partial A) \mathrm{d} x .
$$

Proof. We follow [14, proposition 4.5] with minor modifications and we give the details for the convenience of the reader. Define

$$
E:=\{x \in B \Delta A: d(x, \partial A) \leqslant \ell\}, \quad F:=\left\{x \in B \Delta A: d\left(x, \partial A_{j}\right) \geqslant \ell\right\} .
$$

By the Chebyshev inequality,

$$
|F| \leqslant \frac{1}{\ell} \int_{F} d(x, \partial A) \mathrm{d} x \leqslant \frac{1}{\ell} \int_{A \Delta B} d(x, \partial A) \mathrm{d} x .
$$

Let us estimate $|E|$. By a covering argument, one can find a finite family of disjoint balls $\left\{B_{\ell}\left(x_{k}\right)\right\} \quad x_{k} \in \partial A$, such that $E$ is covered by the family $\left\{B_{5 \ell}\left(x_{k}\right)\right\}_{k=1}^{m}$. If 
$\ell \geqslant r_{0}$, by (3.21) and the disjointness of $\left\{B_{\ell}\left(x_{k}\right)\right\}$ (and hence of $\left\{B_{r_{0}}\left(x_{k}\right)\right\}$ ),

$$
\begin{aligned}
|E| & \leqslant \sum_{k=1}^{m} \omega_{n}(5 \ell)^{n}=\frac{5^{n} \omega_{n} \ell^{n}}{\theta r_{0}^{n-1}} \sum_{k=1}^{m} \theta r_{0}^{n-1} \leqslant \frac{5^{n} \omega_{n} \ell^{n}}{\theta r_{0}^{n-1}} \sum_{k=1}^{m} P\left(A_{j}, B_{r_{0}}\left(x_{k}\right)\right) \\
& \leqslant \frac{5^{n} \omega_{n} \ell^{n}}{\theta r_{0}^{n-1}} P\left(A_{j}, \bigcup_{k=1}^{m} B_{r_{0}}\left(x_{k}\right)\right) \leqslant \frac{5^{n} \omega_{n}}{\theta}\left(\frac{\ell}{r_{0}}\right)^{n-1} P\left(A_{j}\right) \ell .
\end{aligned}
$$

Analogously, if $\ell<r_{0}$, then

$$
|E| \leqslant \frac{5^{n} \omega_{n}}{\theta} P\left(A_{j}\right) \ell
$$

Now (3.22) follows from the inequality $|B \Delta A| \leqslant|E|+|F|$ and estimates for $|A|$ and $|B|$.

\section{Existence of GMM for bounded partitions}

Given a norm $\psi$ in $\mathbb{R}^{n}$ and $E, F \subseteq \mathbb{R}^{n}$ set

$$
\bar{\sigma}_{\psi}(E, F):=\int_{E \Delta F} d_{\psi}(x, \partial F) \mathrm{d} x .
$$

Note that $\bar{\sigma}_{\psi}(E, F)=0$ if $|E \Delta F|=0$ whereas $\bar{\sigma}_{\psi}(E, F)=+\infty$ if $\partial F=\emptyset$ and $|E \Delta F|>0$. Moreover, $X, Y \subseteq \mathbb{R}^{n}$ are measurable and $\partial Y \neq \emptyset$,

$$
\begin{aligned}
\int_{X \Delta Y} d_{\psi}(x, \partial Y) \mathrm{d} x= & \int_{X} \widetilde{d}_{\psi}(x, \partial Y) \mathrm{d} x \\
& -\int_{Y} \widetilde{d}_{\psi}(x, \partial Y) \mathrm{d} x \quad \text { if } X \cap Y \text { is bounded, } \\
\int_{X \Delta Y} d_{\psi}(x, \partial Y) \mathrm{d} x= & \int_{Y^{c}} \widetilde{d}_{\psi}(x, \partial Y) \mathrm{d} x \\
& -\int_{X^{c}} \widetilde{d}_{\psi}(x, \partial Y) \mathrm{d} x \quad \text { if } X^{c} \cap Y^{c} \text { is bounded. }
\end{aligned}
$$

Given a family $\Psi:=\left\{\psi_{1}, \ldots, \psi_{N+1}\right\}$ of norms $\psi_{i}$ in $\mathbb{R}^{n}$, and $\mathcal{A}, \mathcal{B} \in \mathbb{P}_{b}(N+1)$, we set

$$
\sigma_{\Psi}(\mathcal{A}, \mathcal{B}):=\sum_{i=1}^{N+1} \bar{\sigma}_{\psi_{i}}\left(A_{i}, B_{i}\right),
$$

where $N+1 \geqslant 2$. In the literature $\Psi$ is called the set of mobilities. Since $N$ is finite, there exist $0<c_{\Psi} \leqslant C_{\Psi}<+\infty$ such that

$$
c_{\Psi} \leqslant \psi_{i}(\nu) \leqslant C_{\Psi}, \quad i=1, \ldots, N+1, \quad \nu \in \mathbb{S}^{n-1} .
$$

Observe that for every $\mathcal{B} \in \mathbb{P}_{b}(N+1)$ the map $\sigma_{\Psi}(\cdot, \mathcal{B})$ is $L^{1}\left(\mathbb{R}^{n}\right)$-lower semicontinuous in $\mathbb{P}_{b}(N+1)$.

Given families $\Phi:=\left\{\phi_{1}, \ldots, \phi_{N+1}\right\}$ of anisotropies and $\Psi:=\left\{\psi_{1}, \ldots, \psi_{N+1}\right\}$ of mobilities, and $\mathbf{H}:=\left(H_{1}, \ldots, H_{N+1}\right)$ of functions $H_{i} \in L_{\text {loc }}^{1}\left(\mathbb{R}^{n}\right), \quad i=$ 
$1, \ldots, N$, and $H_{N+1} \in L^{1}\left(\mathbb{R}^{n}\right)$, consider the functional $\mathfrak{F}: \mathbb{P}_{b}(N+1) \times \mathbb{P}_{b}$ $(N+1) \times[1, \infty) \rightarrow[-\infty, \infty]$

$$
\mathfrak{F}(\mathcal{E}, \mathcal{F}, \lambda)=\operatorname{Per}_{\Phi}(\mathcal{E})+\Upsilon(\mathcal{E})+\lambda \sigma_{\Psi}(\mathcal{E}, \mathcal{F}),
$$

where

$$
\Upsilon(\mathcal{E}):=\sum_{i=1}^{N+1} \int_{E_{i}} H_{i} \mathrm{~d} x .
$$

Note that $\mathfrak{F}(\cdot, \mathcal{F} ; \lambda)$ is well-defined and $L^{1}\left(\mathbb{R}^{n}\right)$-lower semicontinuous in $\mathbb{P}_{b}$ $(N+1)$. Notice that $\Upsilon$ can also be represented as

$$
\Upsilon(\mathcal{E})=\sum_{j=1}^{N} \int_{E_{j}}\left(H_{j}-H_{N+1}\right) \mathrm{d} x+\int_{\mathbb{R}^{n}} H_{N+1} \mathrm{~d} x
$$

The functional $\mathfrak{F}$ is a generalization of the Almgren-Taylor-Wang functional [1] to the case of partitions $[\mathbf{1 4}, \mathbf{2 2}]$ in presence of anisotropies, mobilities and external forces.

The main result of this section is the following, which generalizes $[\mathbf{1 4}$, theorems 4.9 and 5.1] to the anisotropic case with mobilities; recall that $\kappa_{N+1}$ is defined in (3.1).

THEOREM 4.1 Existence of $G M M$. Let $\Phi=\left\{\phi_{1}, \ldots, \phi_{N+1}\right\}$ and $\Psi=$ $\left\{\psi_{1}, \ldots, \psi_{N+1}\right\}$ be families of anisotropies and mobilities, respectively. Suppose that

$$
\kappa_{N+1}<\frac{2 c_{\Phi}}{N}
$$

and $\boldsymbol{H}=\left(H_{1}, \ldots, H_{N+1}\right)$ satisfies

$$
\left\{\begin{array}{l}
H_{i} \in L_{\mathrm{loc}}^{p}\left(\mathbb{R}^{n}\right), i=1, \ldots, N+1, \text { for some } p>n \text { and } H_{N+1} \in L^{1}\left(\mathbb{R}^{n}\right) \\
{[1 m m] \exists R>0 \text { s.t. } H_{i} \geqslant H_{N+1} \text { a.e. in } \mathbb{R}^{n} \backslash B_{R}(0) \text { for } i=1, \ldots, N .}
\end{array}\right.
$$

Then for every $\mathcal{G} \in \mathbb{P}_{b}(N+1), G M M(\mathfrak{F}, \mathcal{G})$ is nonempty. Moreover, there exists a constant $C=C(N, n, \Phi, \Psi, \boldsymbol{H}, \mathcal{G})>0$ such that for any $\mathcal{M} \in G M M(\mathfrak{F}, \mathcal{G})$,

$$
\left|\mathcal{M}(t) \Delta \mathcal{M}\left(t^{\prime}\right)\right| \leqslant C\left|t-t^{\prime}\right|^{\frac{1}{n+1}}, \quad t, t^{\prime}>0,\left|t-t^{\prime}\right|<1
$$

and

$$
\bigcup_{j=1}^{N} M_{j}(t) \subseteq D:=\text { closed convex hull of } \operatorname{co}(\mathcal{G}) \cup B_{R} \quad \forall t \geqslant 0
$$

and $B_{R}$ is not present in (4.6) if $\boldsymbol{H} \equiv 0$. In addition, if $\sum_{j=1}^{N+1}\left|\overline{G_{j}} \backslash G_{j}\right|=0$, then (4.5) holds for any $t, t^{\prime} \geqslant 0$ with $\left|t-t^{\prime}\right|<1$.

Proof. We give only few details of the proof since it can be done following the arguments of the proofs of [14, theorems 4.9 and 5.1]. 
Step 1: Existence of minimizers. Given $\mathcal{A} \in \mathbb{P}_{b}(N+1)$ and $\lambda \geqslant 1$, the problem

$$
\inf _{\mathcal{B} \in \mathbb{P}_{b}(N+1)} \mathfrak{F}(\mathcal{B}, \mathcal{A} ; \lambda)
$$

has a solution. Moreover, every minimizer $\mathcal{A}(\lambda)=\left(A_{1}(\lambda), \ldots, A_{N+1}(\lambda)\right)$ satisfies the bound

$$
\bigcup_{i=1}^{N} A_{i}(\lambda) \subseteq \text { closed convex hull of } \operatorname{co}(\mathcal{A}) \cup B_{R}(0)
$$

We omit the proof since it is proven along the same lines as [14, theorem 4.2] using the anisotropic comparison theorem with convex sets ${ }^{1}$ and the inequality $d_{\psi}\left(\cdot, E_{0}\right)>0$ in any $F \subset \mathbb{R}^{n} \backslash \overline{E_{0}}$.

Step 2: Density estimates for minimizers. Let $\mathcal{A} \in \mathbb{P}_{b}(N+1)$ satisfy $\operatorname{co}(\mathcal{A}) \subset D$ and set

$$
\Lambda_{1}:=\lambda \max _{1 \leqslant j \leqslant N+1}\left(\operatorname{diam}_{\psi_{j}} D+2\right), \quad \Lambda_{2}:=N^{1 / p} \max _{1 \leqslant j \leqslant N}\left\|H_{j}-H_{N+1}\right\|_{L^{p}\left(D_{1}\right)},
$$

where $D_{1}:=\left\{x \in \mathbb{R}^{n}: d(x, D) \leqslant 1\right\}$. Let $\lambda \geqslant 1$ and $\mathcal{A}(\lambda) \in \mathbb{P}_{b}(N+1)$ be a minimizer of $\mathfrak{F}(\cdot, \mathcal{A} ; \lambda)$. Then for every $i \in\{1, \ldots, N+1\}$ either $\partial A_{i}(\lambda)$ is empty or for any $x \in \partial A_{i}(\lambda)$ and

$$
r \in\left(0, \min \left\{1,\left(\frac{c_{\Phi}}{N}-\frac{\kappa_{N+1}}{2}\right) \frac{n}{4 \Lambda_{1}},\left[\left(\frac{c_{\Phi}}{N}-\frac{\kappa_{N+1}}{2}\right) \frac{n \omega_{n}^{1 / p}}{2^{2-1 / p} \Lambda_{2}}\right]^{p /(p-n)}\right\}\right]
$$

one has

$$
\left(\frac{2 c_{\Phi}-N \kappa_{N+1}}{2 c_{\Phi}+2 N C_{\Phi}-N \kappa_{N+1}}\right)^{n} \leqslant \frac{\left|A_{i}(\lambda) \cap B_{r}(x)\right|}{\left|B_{r}(x)\right|} \leqslant 1-\left(\frac{c_{\Phi}}{2\left(c_{\Phi}+C_{\Phi}\right)}\right)^{n}
$$

and

$$
c^{\Phi} \leqslant \frac{P\left(A_{i}(\lambda), B_{r}(x)\right)}{r^{n-1}} \leqslant\left(\frac{C_{\Phi}}{c_{\Phi}}+\frac{1}{2}\right) n \omega_{n}
$$

where $\kappa_{N+1}$ is given by (3.1) and

$$
c^{\Phi}=c^{\Phi}(N, n):=\frac{n \omega_{n}\left(2^{1 / n}-1\right)}{2^{1+1 / n}}\left(\frac{2 c_{\Phi}-N \kappa_{N+1}}{2 c_{\Phi}+2 N C_{\Phi}-N \kappa_{N+1}}\right)^{n-1} .
$$

The proof is analogous to the the proof of $[\mathbf{1 4}$, theorem 4.6$]$ : we only show that

$$
\mathcal{A}(\lambda) \text { is a }\left(\Phi, \Lambda_{1}, \Lambda_{2}, 1,1-1 / p\right) \text {-minimizer }
$$

${ }^{1}$ If $E \in B V\left(\mathbb{R}^{n} ;\{0,1\}\right)$, then $P_{\phi}(E) \geqslant P_{\phi}(E \cap C)$ for every anisotropy $\phi$ and every closed convex set $C \subset \mathbb{R}^{n}$. 
and hence (4.9)-(4.10) follow from theorem 3.2. Let $\mathcal{C} \in \mathbb{P}_{b}(N+1)$ be such that $\mathcal{C} \Delta \mathcal{A}(\lambda) \subset \subset B_{\rho}(x)$ with $\rho \in(0,1)$. By the minimality of $\mathcal{A}(\lambda)$,

$$
\begin{aligned}
\operatorname{Per}_{\Phi}\left(\mathcal{A}(\lambda), B_{\rho}(x)\right) \leqslant & \operatorname{Per}_{\Phi}\left(\mathcal{C}, B_{\rho}(x)\right)+\lambda \sum_{j=1}^{N+1} \int_{C_{j} \Delta A_{j}(\lambda)} d_{\psi_{j}}\left(x, \partial A_{j}\right) \mathrm{d} x \\
& +\sum_{j=1}^{N} \int_{C_{j} \Delta A_{j}(\lambda)}\left|H_{j}-H_{N+1}\right| \mathrm{d} x .
\end{aligned}
$$

By step $1, \operatorname{co}(\mathcal{A}(\lambda)) \subseteq D$, thus

$$
d_{\psi_{j}}\left(z, \partial A_{j}\right) \leqslant \operatorname{diam}_{\psi_{j}} D+2 \rho \text { for all } j=1, \ldots, N+1 \text { and } z \in \mathcal{C} \Delta \mathcal{A}(\lambda),
$$

where $\operatorname{diam}_{\psi_{j}}$ is the $\psi_{j}$-diameter of a set. Then, since $\mathcal{C} \Delta \mathcal{A}(\lambda) \subset D_{1}$,

$$
\sum_{j=1}^{N+1} \int_{C_{j} \Delta A_{j}(\lambda)} d_{\psi_{j}}\left(x, \partial A_{j}\right) \mathrm{d} x \leqslant \max _{1 \leqslant j \leqslant N+1}\left(\operatorname{diam}_{\psi_{j}} D+2\right)|\mathcal{C} \Delta \mathcal{A}(\lambda)|
$$

and

$$
\begin{aligned}
\sum_{j=1}^{N} \int_{C_{j} \Delta A_{j}(\lambda)}\left|H_{j}-H_{N+1}\right| \mathrm{d} x & \leqslant \sum_{j=1}^{N}\left|C_{j} \Delta A_{j}(\lambda)\right|^{1-1 / p}\left\|H_{j}-H_{N+1}\right\|_{L^{p}\left(D_{1}\right)} \\
& \leqslant N^{1 / p} \max _{1 \leqslant j \leqslant N}\left\|H_{j}-H_{N+1}\right\|_{L^{p}\left(D_{1}\right)}|\mathcal{C} \Delta \mathcal{A}(\lambda)|^{1-1 / p}
\end{aligned}
$$

Thus,

$$
\operatorname{Per}_{\Phi}\left(\mathcal{A}(\lambda), B_{\rho}(x)\right) \leqslant \operatorname{Per}_{\Phi}\left(\mathcal{C}, B_{\rho}(x)\right)+\Lambda_{1}|\mathcal{C} \Delta \mathcal{A}(\lambda)|+\Lambda_{2}|\mathcal{C} \Delta \mathcal{A}(\lambda)|^{1-1 / p} .
$$

Step 3: Existence of GMM. Given $\lambda \geqslant 1$ and $k \in \mathbb{N}_{0}$ we define $\mathcal{G}(\lambda, k)$ recursively as: $\mathcal{G}(\lambda, 0)=\mathcal{G}$ and

$$
\mathfrak{F}(\mathcal{G}(\lambda, k), \mathcal{G}(\lambda, k-1), \lambda)=\min _{\mathcal{A} \in \mathbb{P}_{b}(N+1)} \mathfrak{F}(\mathcal{A}, \mathcal{G}(\lambda, k-1), \lambda) .
$$

Since $\mathfrak{F}(\mathcal{G}(\lambda, k), \mathcal{G}(\lambda, k-1), \lambda) \leqslant \mathfrak{F}(\mathcal{G}(\lambda, k-1), \mathcal{G}(\lambda, k-1), \lambda)$, we have

$$
\begin{aligned}
\operatorname{Per}_{\Phi}(\mathcal{G}(\lambda, k))+\Upsilon(\mathcal{G}(\lambda, k))+ & \lambda \sigma_{\Psi}(\mathcal{G}(\lambda, k), \mathcal{G}(\lambda, k-1)) \\
& \leqslant \operatorname{Per}_{\Phi}(\mathcal{G}(\lambda, k-1))+\Upsilon(\mathcal{G}(\lambda, k-1)) .
\end{aligned}
$$

Thus, the map $k \in \mathbb{N}_{0} \mapsto \operatorname{Per}_{\Phi}(\mathcal{G}(\lambda, k))+\Upsilon(\mathcal{G}(\lambda, k))$ is nonincreasing for any $\lambda \geqslant$ 1. In particular,

$$
\begin{aligned}
\operatorname{Per}_{\Phi}(\mathcal{G}(\lambda, k)) & \leqslant \operatorname{Per}_{\Phi}(\mathcal{G}(\lambda, 0))+\sum_{j=1}^{N} \int_{G_{j}(\lambda, k) \Delta G_{j}(\lambda, 0)}\left|H_{j}-H_{N+1}\right| \mathrm{d} x \\
& \leqslant \operatorname{Per}_{\Phi}(\mathcal{G})+\sum_{j=1}^{N}\left\|H_{j}-H_{N+1}\right\|_{L^{1}(D)}=: \mu_{0}, \quad k \geqslant 0
\end{aligned}
$$


and

$$
\bigcup_{j=1}^{N} G_{j}(\lambda, k) \subseteq D \text { for all } \lambda \geqslant 1 \text {, and } k \geqslant 0 .
$$

Fix $t, t^{\prime}>0$ with $0<t-t^{\prime}<1$. Let $\lambda>1$ be so large (depending on $t, t^{\prime}, n$, $N, \quad \mathbf{H}, \Psi$ and $\Phi)$ that setting $k_{0}=\left[\lambda t^{\prime}\right], m_{0}=[\lambda t]$, one has $m_{0} \geqslant k_{0}+3 \geqslant 4$ and

$$
r_{\lambda}:=\min \left\{1,\left(\frac{c_{\Phi}}{N}-\frac{\kappa_{N+1}}{2}\right) \frac{n}{4 \Lambda_{1}},\left[\left(\frac{c_{\Phi}}{N}-\frac{\kappa_{N+1}}{2}\right) \frac{n \omega_{n}^{1 / p}}{2^{2-1 / p} \Lambda_{2}}\right]^{p /(p-n)}\right\}=\frac{\gamma}{\lambda},
$$

where $\Lambda_{1}$ and $\Lambda_{2}$ are given in (4.7), and recalling (4.3),

$$
\gamma:=\left(\frac{c_{\Phi}}{N}-\frac{\kappa_{N+1}}{2}\right) \frac{n}{4 \max _{1 \leqslant j \leqslant N+1}\left(\operatorname{diam}_{\psi_{j}} D+2\right)}>0 .
$$

By (4.10) for such $\lambda$ and for any $k \geqslant 1$ any minimizer $\mathcal{G}(\lambda, k)$ satisfies

$$
P\left(G_{j}(\lambda, k), B_{r}(x)\right) \geqslant c^{\Phi} r^{n-1}
$$

for any $x \in \partial G_{j}(\lambda, k)$ and $r \in\left(0, r_{\lambda}\right)$ provided $\partial G_{j}(\lambda, k)$ is nonempty. Therefore, by proposition 3.3 applied with $r_{0}=r_{\lambda}, \quad \theta=c^{\Phi}, \quad A=G_{j}(\lambda, k-1), \quad B=$ $G_{j}(\lambda, k)$ and $\ell=r_{\lambda}\left|t-t^{\prime}\right|^{-1 /(n+1)}>r_{\lambda}$, for any $j \in\{1, \ldots, N+1\}$ and $k \in$ $\left\{k_{0}+1, \ldots, m_{0}\right\}$, we have

$$
\begin{aligned}
|\mathcal{G}(\lambda, k-1) \Delta \mathcal{G}(\lambda, k)| \leqslant & \frac{5^{n} \omega_{n} \gamma}{\lambda c^{\Phi}\left|t-t^{\prime}\right|^{n / n+1}} \operatorname{Per}(\mathcal{G}(\lambda, k-1)) \\
& +\frac{\lambda\left|t-t^{\prime}\right|^{1 / n+1}}{\gamma} \sigma(\mathcal{G}(\lambda, k), \mathcal{G}(\lambda, k-1)) .
\end{aligned}
$$

Now the bounds (2.5), (4.1) and (4.11) imply

$$
\begin{aligned}
& |\mathcal{G}(\lambda, k-1) \Delta \mathcal{G}(\lambda, k)| \leqslant \frac{5^{n} \omega_{n} \gamma}{\lambda c_{\Phi} c^{\Phi}\left|t-t^{\prime}\right|^{n / n+1}} \operatorname{Per}_{\Phi}(\mathcal{G}(\lambda, k-1)) \\
& +\frac{\left|t-t^{\prime}\right|^{1 / n+1}}{\gamma c_{\Psi}}\left(\operatorname{Per}_{\Phi}(\mathcal{G}(\lambda, k-1))+\Upsilon(\mathcal{G}(\lambda, k-1))-\operatorname{Per}_{\Phi}(\mathcal{G}(\lambda, k)-\Upsilon(\mathcal{G}(\lambda, k))) .\right.
\end{aligned}
$$

Summing this inequality in $k \in\left\{k_{0}+1, \ldots, m_{0}\right\}$, we obtain

$$
\begin{aligned}
& \left|\mathcal{G}(\lambda,[\lambda t]) \Delta \mathcal{G}\left(\lambda,\left[\lambda t^{\prime}\right]\right)\right| \leqslant \sum_{k=k_{0}+1}^{m_{0}}|\mathcal{G}(\lambda, k-1) \Delta \mathcal{G}(\lambda, k)| \\
& \leqslant \frac{5^{n} \omega_{n} \gamma}{\lambda c_{\Phi} c^{\Phi}\left|t-t^{\prime}\right|^{\frac{n}{n+1}}} \sum_{k=k_{0}+1}^{m_{0}} \operatorname{Per}_{\Phi}(\mathcal{G}(\lambda, k-1)) \\
& \quad+\frac{\left|t-t^{\prime}\right|^{\frac{1}{n+1}}}{\gamma c_{\Psi}}\left(\operatorname{Per}_{\Phi}\left(\mathcal{G}\left(\lambda, k_{0}\right)\right)+\Upsilon\left(\mathcal{G}\left(\lambda, k_{0}\right)\right)-\operatorname{Per}_{\Phi}\left(\mathcal{G}\left(\lambda, m_{0}\right)-\Upsilon\left(\mathcal{G}\left(\lambda, m_{0}\right)\right)\right) .\right.
\end{aligned}
$$


By (4.12)

$$
\sum_{k=k_{0}+1}^{m_{0}} \operatorname{Per}_{\Phi}(\mathcal{G}(\lambda, k-1)) \leqslant \mu_{0}\left(m_{0}-k_{0}\right) \leqslant \lambda \mu_{0}\left(\left|t-t^{\prime}\right|+\frac{1}{\lambda}\right)
$$

and

$$
\begin{aligned}
\operatorname{Per}_{\Phi}\left(\mathcal{G}\left(\lambda, k_{0}\right)\right)+\Upsilon\left(\mathcal{G}\left(\lambda, k_{0}\right)\right) & -\operatorname{Per}_{\Phi}\left(\mathcal{G}\left(\lambda, m_{0}\right)\right)-\Upsilon\left(\mathcal{G}\left(\lambda, m_{0}\right)\right) \\
& \leqslant \operatorname{Per}_{\Phi}\left(\mathcal{G}\left(\lambda, k_{0}\right)\right)+\sum_{j=1}^{N}\left\|H_{j}-H_{N+1}\right\|_{L^{1}(D)} \leqslant 2 \mu_{0} .
\end{aligned}
$$

Thus, from (4.14) we get

$$
\left|\mathcal{G}(\lambda,[\lambda t]) \Delta \mathcal{G}\left(\lambda,\left[\lambda t^{\prime}\right]\right)\right| \leqslant C\left|t-t^{\prime}\right|^{\frac{1}{n+1}}+\widetilde{C}\left|t-t^{\prime}\right|^{-\frac{n}{n+1}} \lambda^{-1}
$$

where

$$
C:=\frac{5^{n} \omega_{n} \gamma \mu_{0}}{c_{\Phi} c^{\Phi}}+\frac{2 \mu_{0}}{\gamma c_{\Psi}} \text { and } \quad \widetilde{C}:=\frac{5^{n} \omega_{n} \gamma \mu_{0}}{c_{\Phi} c^{\Phi}} .
$$

The remaining part of the proof is as the proofs of [14, theorems 4.9 and 5.1]. We note here that if $\mathcal{G} \in \mathbb{P}_{b}(N+1)$ satisfies $\sum_{j=1}^{N+1}\left|\overline{G_{j}} \backslash G_{j}\right|=0$, then $1 /(n+1)$ Hölderianity of GMM at time $t=0$ follows from the relations

$$
\begin{array}{r}
\lim _{\lambda \rightarrow+\infty}|\mathcal{G}(\lambda, 1) \Delta \mathcal{G}|=0, \quad \lim _{\lambda \rightarrow+\infty} \operatorname{Per}_{\Phi}(\mathcal{G}(\lambda, 1))=\operatorname{Per}_{\Phi}(\mathcal{G}), \\
\lim _{\lambda \rightarrow+\infty} \lambda \sigma_{\Psi}(\mathcal{G}(\lambda, 1), \mathcal{G})=0, \quad \lim _{\lambda \rightarrow+\infty} \Upsilon(\mathcal{G}(\lambda, 1))=\Upsilon(\mathcal{G})
\end{array}
$$

whose proofs can be done following the arguments of [14, proposition 4.5].

\subsection{Two-phase case}

When $N=1$, repeating the arguments of $[\mathbf{4 2}]$ in our more general setting, we can improve the Hölder exponent of GMM to $1 / 2$ without any restriction on the anisotropies.

Theorem 4.2. Let $\Phi=\left(\phi_{1}, \phi_{2}\right)$ and $\Psi=\left(\psi_{1}, \psi_{2}\right)$, and assume that $\boldsymbol{H}=\left(H_{1}\right.$, $\left.H_{2}\right)$ satisfies (4.4) with $N=1$. Then for every $\mathcal{G} \in \mathbb{P}_{b}(2), \quad \operatorname{GMM}(\mathfrak{F}, \mathcal{G})$ is nonempty. Moreover, there exists a constant $C=C\left(n, \Phi, \Psi, \boldsymbol{H}, \operatorname{Per}_{\Phi}(\mathcal{G})\right)>0$ such that for any $\mathcal{N} \in G M M(\mathfrak{F}, \mathcal{G})$,

$$
\left|\mathcal{N}(t) \Delta \mathcal{N}\left(t^{\prime}\right)\right| \leqslant C\left|t-t^{\prime}\right|^{1 / 2}, \quad t, t^{\prime}>0,\left|t-t^{\prime}\right|<1
$$

and

$$
N_{1}(t) \subseteq \text { closed convex hull of } G_{1} \cup B_{R} \quad \forall t \geqslant 0 .
$$

In addition, if $\left|\overline{G_{1}} \backslash G_{1}\right|=0$, then (4.16) holds for any $t, t^{\prime} \geqslant 0$ with $\left|t-t^{\prime}\right|<1$. 
The proof runs along the same lines of theorem 4.1 with however an improved bound for the radii in the proof of the density estimates, see (4.30) below. We need to make a detailed proof since this will be used in the proof of theorem 5.1.

Proof. Letting $\phi:=\phi_{1}+\phi_{2}, \quad H:=H_{1}-H_{2}$, and

$$
d_{\psi}^{E}(\cdot):=d_{\psi_{1}}(\cdot, \partial E)+d_{\psi_{2}}(\cdot, \partial E), \quad \widetilde{d}_{\psi}^{E}(\cdot):=\widetilde{d}_{\psi_{1}}(\cdot, \partial E)+\widetilde{d}_{\psi_{2}}(\cdot, \partial E),
$$

we have

$$
\begin{aligned}
& \mathfrak{F}(\mathcal{A}, \mathcal{B}, \lambda)-\int_{\mathbb{R}^{n}} H_{2} \mathrm{~d} x=P_{\phi}\left(A_{1}\right)+\int_{A_{1}} H \mathrm{~d} x+\lambda \int_{A_{1} \Delta B_{1}} d_{\psi}^{B_{1}} \mathrm{~d} x \\
= & P_{\phi}\left(A_{1}\right)+\int_{A_{1}} H \mathrm{~d} x+\lambda \int_{A_{1}} \widetilde{d}_{\psi}^{B_{1}} \mathrm{~d} x-\lambda \int_{B_{1}} \widetilde{d}_{\psi}^{B_{1}} \mathrm{~d} x=: \mathfrak{F}_{2}\left(A_{1}, B_{1}, \lambda\right) .
\end{aligned}
$$

Therefore, it suffices to show that for any bounded $G \in B V\left(\mathbb{R}^{n} ;\{0,1\}\right)$, $\operatorname{GMM}\left(\mathfrak{F}_{2}, G\right)$ is nonempty and there exists $C_{0}:=C_{0}\left(n, \Phi, \Psi, H, P_{\phi}(G)\right)$ such that for any $L \in G M M\left(\mathfrak{F}_{2}, G\right)$

$$
|L(t) \Delta L(s)| \leqslant C_{0}\left|t-t^{\prime}\right|^{1 / 2}, \quad t, t^{\prime}>0, \quad\left|t-t^{\prime}\right|<1
$$

and

$$
L(t) \subseteq D:=\operatorname{co}\left(G \cup B_{R}\right), \quad t \geqslant 0 .
$$

Note that, except for the presence of $\int_{A_{1}} H \mathrm{~d} x, \mathfrak{F}_{2}$ is of the form of the AlmgrenTaylor-Wang functional.

We divide the proof into five steps.

Step 1: Existence of minimizers. Let $E_{0} \in B V\left(\mathbb{R}^{n} ;\{0,1\}\right)$ be such that $E_{0} \subset D$. Since $\phi$ is a norm, $\widetilde{d}_{\psi}^{E_{0}} \geqslant 0$ in $\mathbb{R}^{n} \backslash E_{0}$ and $H \geqslant 0$ in $\mathbb{R}^{n} \backslash D$, as in the Euclidean two-phase case (see e.g. [3]) we can use the comparison theorem with the convex set $D$ to establish the existence of a minimizer of $\mathfrak{F}_{2}\left(\cdot, E_{0}, \lambda\right)$ and also that every minimizer $E_{\lambda}$ satisfies $E_{\lambda} \subseteq D$.

Step 2: Unconstrained density estimates for minimizers. Let $E_{\lambda}$ minimize $\mathfrak{F}_{2}\left(\cdot, E_{0}, \lambda\right)$ and $x_{0} \in E_{\lambda} \Delta E_{0}$ be such that $d\left(x_{0}, \partial E_{0}\right) \geqslant r_{1}$ for some $r_{1}>0$ satisfying

$$
w_{n}^{1 / n-1 / p} r_{1}^{1-n / p}\left(\int_{D}|H|^{p} \mathrm{~d} x\right)^{1 / p}<c_{\Phi} n \omega_{n}^{1 / n}
$$

Notice that there are no restrictions on $r_{1}>0$; in addition $x_{0}$ need not to be on $\partial E_{\lambda}$. Let us show that

(a) if $x_{0} \in E_{\lambda} \backslash E_{0}$, then

$$
\frac{\left|E_{\lambda} \cap B_{r}\left(x_{0}\right)\right|}{\left|B_{r}\left(x_{0}\right)\right|} \geqslant\left(\frac{c_{\Phi}}{4 C_{\Phi}}\right)^{n} \quad \text { for any } r \in\left(0, r_{1}\right)
$$

(b) if $x_{0} \in E_{0} \backslash E_{\lambda}$, then

$$
\frac{\left|B_{r}\left(x_{0}\right) \backslash E_{\lambda}\right|}{\left|B_{r}\left(x_{0}\right)\right|} \geqslant\left(\frac{c_{\Phi}}{4 C_{\Phi}}\right)^{n} \text { for any } r \in\left(0, r_{1}\right) .
$$


We prove only (a), since the proof of (b) is similar. For shortness we write $B_{r}:=$ $B_{r}\left(x_{0}\right)$. Fix any $r \in\left(0, r_{1}\right)$ such that

$$
\mathcal{H}^{n-1}\left(\partial^{*} E_{\lambda} \cap \partial B_{r}\right)=0 .
$$

By the minimality of $E_{\lambda}$ we have $\mathfrak{F}_{2}\left(E_{\lambda}, E_{0}, \lambda\right) \leqslant \mathfrak{F}_{2}\left(E_{\lambda} \backslash B_{r}, E_{0}, \lambda\right)$ so that

$$
P_{\phi}\left(E_{\lambda}, B_{s}\right)+\lambda \int_{E_{\lambda} \cap B_{r}} \widetilde{d}_{\psi}^{E_{0}} \mathrm{~d} x \leqslant P_{\phi}\left(E_{\lambda} \backslash B_{r}, B_{s}\right)+\int_{E_{\lambda} \cap B_{r}}|H| \mathrm{d} x
$$

for any $s>r$. The choice of $x_{0}$ and the definition of $\widetilde{d}_{\psi}^{E_{0}}$ imply $\widetilde{d}_{\psi}^{E_{0}} \geqslant 0$ in $B_{r_{1}}$ and hence using (4.21) and (3.12) (applied with $\phi_{j}=\phi$ and $A_{j}=E_{\lambda}$ ) and the inclusion $\left(E_{\lambda} \backslash B_{r}\right) \Delta E_{\lambda} \subset B_{r}$, from (4.22) we get

$$
P_{\phi}\left(E_{\lambda} \cap B_{r}\right) \leqslant 2 \int_{E_{\lambda} \cap \partial B_{r}} \phi\left(\nu_{B_{r}}\right) \mathrm{d} \mathcal{H}^{n-1}+\int_{E_{\lambda} \cap B_{r}}|H| \mathrm{d} x .
$$

The definition of $\phi,(2.5)$, the isoperimetric inequality and the Hölder inequality yield

$$
\begin{aligned}
2 c_{\Phi} n \omega_{n}^{1 / n}\left|E_{\lambda} \cap B_{r}\right|^{(n-1 / n} \leqslant & 4 C_{\Phi} \mathcal{H}^{n-1}\left(E_{\lambda} \cap \partial B_{r}\right) \\
& +\left|E_{\lambda} \cap B_{r}\right|^{1-\frac{1}{p}}\left(\int_{E_{\lambda} \cap B_{r}}|H|^{p} \mathrm{~d} x\right)^{1 / p} .
\end{aligned}
$$

Recall by step 1 that $E_{\lambda} \subseteq D$. Thus from the inequality

$$
\left|E_{\lambda} \cap B_{r}\right|^{1-1 / p} \leqslant\left|E_{\lambda} \cap B_{r}\right|^{(n-1 / n}\left|B_{r}\right|^{1 / n-1 / p}=\omega_{n}^{1 / n-1 / p} r^{1-n / p}\left|E_{\lambda} \cap B_{r}\right|^{(n-1 / n}
$$

and (4.20), it follows that

$$
\left|E_{\lambda} \cap B_{r}\right|^{1-1 / p}\left(\int_{E_{\lambda} \cap B_{r}}|H|^{p} \mathrm{~d} x\right)^{1 / p} \leqslant c_{\Phi} n \omega_{n}^{1 / n}\left|E_{\lambda} \cap B_{r}\right|^{(n-1) / n},
$$

and therefore, from (4.24) we deduce

$$
c_{\Phi} n \omega_{n}^{1 / n}\left|E_{\lambda} \cap B_{r}\right|^{(n-1) / n} \leqslant 4 C_{\Phi} \mathcal{H}^{n-1}\left(E_{\lambda} \cap \partial B_{r}\right) .
$$

Now integrating we get

$$
\frac{\left|E_{\lambda} \cap B_{r}\right|}{\left|B_{r}\right|} \geqslant\left(\frac{c_{\Phi}}{4 C_{\Phi}}\right)^{n}
$$

for any $r \in\left(0, r_{1}\right)$.

The next step is valid in the two-phase case. We miss the proof of a similar statement in the multiphase case because we are not able to prove the analogue of step $2^{2}$.

${ }^{2}$ In the multiphase case we miss the analogue of (4.23), that was obtained neglecting the term $\int_{E_{\lambda} \cap B_{r}} \widetilde{d}_{\psi}^{E_{0}} \mathrm{~d} x$ in (4.22). For instance, in the planar 4 -phase, at a triple junction involving $\phi_{1}, \phi_{2}, \phi_{3}$ and surrounded by the fourth phase having $\phi_{4}$ as surface tension, it is conceivable that, if $\phi_{1}, \phi_{2}, \phi_{3}$ are quite large compared to $\phi_{4}$, then around the triple point, the fourth phase appears after one minimization step. 
We essentially follow the arguments of $[\mathbf{4 2}, \mathbf{4 8}]$. Let

$$
C_{1}=C_{1}(n, \Phi, \Psi):=8 C_{\Psi}\left(\frac{\left(4 C_{\Phi}\right)^{n+1} n}{2 c_{\Psi} c_{\Phi}^{n}}\right)^{1 / 2}
$$

and

$$
C_{2}=C_{2}(n, \Phi, \Psi, H, p):=\left(n c_{\Phi}\right)^{2 p /(n-p)}\left(\frac{C_{1}}{2 C_{\Psi}}\right)^{2}\left(\frac{1}{\omega_{n}} \int_{D}|H|^{p} \mathrm{~d} x\right)^{2 /(p-n)} .
$$

Step 3: $L^{\infty}$-bound for minimizers. For any $\lambda>C_{2}$, if $E_{\lambda}$ minimizes $\mathfrak{F}_{2}\left(\cdot, E_{0}, \lambda\right)$ then

$$
\sup _{x \in E_{\lambda} \Delta E_{0}} d_{\psi}^{E_{0}}(x) \leqslant C_{1} \lambda^{-1 / 2} .
$$

Assume by contradiction that there exist $\lambda>C_{2}$ and $x_{0} \in E_{\lambda} \Delta E_{0}$ such that $d_{\psi}^{E_{0}}\left(x_{0}\right)>C_{1} \lambda^{-1 / 2}$. Then from (4.1) we get $d\left(x_{0}, \partial E_{0}\right)>C_{1} / 2 C_{\Psi} \lambda^{-1 / 2}$. Since $\lambda>C_{2}$, we can choose $\epsilon>0$ such that $r_{1}:=2 r=d\left(x_{0}, \partial E_{0}\right)>\left(C_{1} / 2 C_{\Psi}+\right.$ $\epsilon) \lambda^{-1 / 2}$ satisfies (4.20), where for shortness we drop the dependence of $r$ on $n$, $\lambda, \epsilon, \Phi$ and $\Psi$. Setting $B_{r}:=B_{r}\left(x_{0}\right)$, without loss of generality we also suppose that (4.21) holds. First we assume $x_{0} \in E_{\lambda} \backslash E_{0}$. Then the minimality of $E_{\lambda}$ implies $\mathfrak{F}_{2}\left(E_{\lambda}, E_{0}, \lambda\right) \leqslant \mathfrak{F}_{2}\left(E_{\lambda} \backslash B_{r}, E_{0}, \lambda\right)$ so that, similarly to (4.23),

$$
P_{\phi}\left(E_{\lambda} \cap B_{r}\right)+\lambda \int_{E_{\lambda} \cap B_{r}} \widetilde{d}_{\psi}^{E_{0}} \mathrm{~d} x \leqslant 2 \int_{E_{\lambda} \cap \partial B_{r}} \phi\left(\nu_{B_{r}}\right) \mathrm{d} \mathcal{H}^{n-1}+\int_{E_{\lambda} \cap B_{r}}|H| \mathrm{d} x .
$$

By the Hölder inequality, the inclusion $E_{\lambda} \subset D$ and (4.20),

$$
\begin{aligned}
\int_{E_{\lambda} \cap B_{r}}|H| \mathrm{d} x & \leqslant\left|E_{\lambda} \cap B_{r}\right|^{1-1 / p}\left(\int_{E_{\lambda} \cap B_{r}}|H|^{p} \mathrm{~d} x\right)^{1 / p} \\
& \leqslant \omega_{n}^{1 / n-1 / p} r^{1-n / p}\left(\int_{D}|H|^{p} \mathrm{~d} x\right)^{1 / p}\left|E_{\lambda} \cap B_{r}\right|^{(n-1) / n}, \\
& <c_{\Phi} n \omega_{n}^{1 / n}\left|E_{\lambda} \cap B_{r}\right|^{(n-1) / n},
\end{aligned}
$$

therefore, by (2.5) and the isoperimetric inequality,

$$
P_{\phi}\left(E_{\lambda} \cap B_{r}\right)>\int_{E_{\lambda} \cap B_{r}}|H| \mathrm{d} x .
$$

This and (4.25) imply

$$
\lambda \int_{E_{\lambda} \cap B_{r}} d_{\psi}^{E_{0}} \mathrm{~d} x<2 \int_{E_{\lambda} \cap \partial B_{r}} \phi\left(\nu_{B_{r}}\right) \mathrm{d} \mathcal{H}^{n-1}
$$

By (4.1), the choice of $x_{0}$ and the definition of $r$ one has $d_{\psi}^{E_{0}} \geqslant c_{\Psi} d\left(\cdot, \partial E_{0}\right) \geqslant 2 c_{\Psi} r$ in $B_{r}$. Thus, from (4.27) and (2.5) we get

$$
2 c_{\Psi} \lambda r\left|E_{\lambda} \cap B_{r}\right|<4 C_{\Phi} \mathcal{H}^{n-1}\left(E_{\lambda} \cap \partial B_{r}\right) .
$$


This, the inequality $\mathcal{H}^{n-1}\left(E_{\lambda} \cap \partial B_{r}\right) \leqslant n \omega_{n} r^{n-1}$ and step 2 (a) imply

$$
2 c_{\Psi} \lambda \omega_{n} r^{n+1}\left(\frac{c_{\Phi}}{4 C_{\Phi}}\right)^{n}<4 C_{\Phi} n \omega_{n} r^{n-1} .
$$

Therefore, by the definition of $C_{1}$ and $r$,

$$
\left(\frac{C_{1}}{8 C_{\Psi}}\right)^{2}=\frac{\left(4 C_{\Phi}\right)^{n+1} n}{2 c_{\Psi} c_{\Phi}^{n}}>\lambda r^{2}>\left(\frac{C_{1}}{8 C_{\Psi}}+\frac{\epsilon}{4}\right)^{2}
$$

a contradiction.

If $x_{0} \in E_{0} \backslash E_{\lambda}$, then we use $\mathfrak{F}_{2}\left(E_{\lambda}, E_{0}, \lambda\right) \leqslant \mathfrak{F}_{2}\left(E_{\lambda} \cup B_{r}, E_{0}, \lambda\right)$ and repeat a similar argument.

Before passing to the next step let us define

$$
\begin{gathered}
C_{3}:=C_{3}(n, \Phi, \Psi)=\frac{2 n c_{\Phi}}{C_{1}+\sqrt{C_{1}^{2}+4 n c_{\Phi} C_{\Psi}}}, \\
C_{4}:=C_{4}(n, \Phi)=\frac{n \omega_{n}\left(2^{1 / n}-1\right)}{2^{n+1 / n}}\left(\frac{c_{\Phi}}{4 C_{\Phi}}\right)^{n-1}
\end{gathered}
$$

and

$$
C_{5}=C_{5}(n, \Phi, \Psi, H, p):=\max \left\{C_{2}, C_{3}^{2}\left(\frac{n c_{\Phi}}{2}\right)^{2 p /(n-p)}\left(\frac{1}{\omega_{n}} \int_{D}|H|^{p} \mathrm{~d} x\right)^{2 /(p-n)}\right\} .
$$

Step 4: Uniform density estimates for minimizers. Given $\lambda>C_{5}$ and a minimizer $E_{\lambda}$ of $\mathfrak{F}_{2}\left(\cdot, E_{0}, \lambda\right)$, following arguments of $[\mathbf{4 2}, \mathbf{4 8}]$ let us show that

$$
\left(\frac{c_{\Phi}}{4 C_{\Phi}}\right)^{n} \leqslant \frac{\left|E_{\lambda} \cap B_{r}(x)\right|}{\left|B_{r}(x)\right|} \leqslant 1-\left(\frac{c_{\Phi}}{4 C_{\Phi}}\right)^{n}
$$

and

$$
C_{4} \leqslant \frac{P\left(E_{\lambda}, B_{r}(x)\right)}{r^{n-1}} \leqslant \frac{2 C_{\Phi}+c_{\Phi}}{2 c_{\Phi}} n \omega_{n}
$$

for any $x \in \partial E_{\lambda}$ and

$$
r \in\left(0, C_{3} \lambda^{-1 / 2}\right)
$$

Since $E_{\lambda}^{(1)}=E_{\lambda}$ and $\overline{\partial^{*} E_{\lambda}}=\partial E_{\lambda}$, we can suppose $x \in \partial^{*} E_{\lambda}$. For any $r$ as in (4.30) and $y \in B_{r}(x)$ one has

$$
d_{\psi}^{E_{0}}(y) \leqslant d_{\psi}^{E_{\lambda}}(y)+\sup _{z \in E_{\lambda} \Delta E_{0}} d_{\psi}^{E_{0}}(z) \leqslant 2 C_{\Psi} r+\sup _{z \in E_{\lambda} \Delta E_{0}} d_{\psi}^{E_{0}}(z)
$$

so that by step 3

$$
d_{\psi}^{E_{0}}(y) \leqslant\left(2 C_{\Psi} C_{3}+C_{1}\right) \lambda^{-1 / 2} .
$$

Let us prove the lower volume density estimate in (4.28). For shortness set $B_{r}:=$ $B_{r}(x)$. Let $r \in\left(0, C_{3} \lambda^{-1 / 2}\right)$ be such that (4.21) holds. As in the proof of step 2, 
from the inequality $\mathfrak{F}_{2}\left(E_{\lambda}, E_{0}, \lambda\right) \leqslant \mathfrak{F}_{2}\left(E_{\lambda} \backslash B_{r}, E_{0}, \lambda\right)$ we get

$$
P_{\phi}\left(E_{\lambda}, B_{r}\right) \leqslant \int_{E_{\lambda} \cap \partial B_{r}} \phi\left(\nu_{B_{r}}\right) \mathrm{d} \mathcal{H}^{n-1}+\lambda \int_{E_{\lambda} \cap B_{r}} d_{\psi}^{E_{0}}(y) \mathrm{d} y+\int_{E_{\lambda} \cap B_{r}}|H| \mathrm{d} y .
$$

By (4.31), the choice of $r$ and the equality

$$
\left(2 C_{\Psi} C_{3}+C_{1}\right) C_{3}=\frac{n c_{\Phi}}{2},
$$

we have

$$
\begin{aligned}
\lambda \int_{E_{\lambda} \cap B_{r}} d_{\psi}^{E_{0}}(y) \mathrm{d} y & \leqslant\left(2 C_{\Psi} C_{3}+C_{1}\right) \omega_{n}^{1 / n} \lambda^{1 / 2} r\left|E_{\lambda} \cap B_{r}\right|^{(n-1) / n} \\
& \leqslant \omega_{n}^{1 / n}\left(2 C_{\Psi} C_{3}+C_{1}\right) C_{3}\left|E_{\lambda} \cap B_{r}\right|^{(n-1) / n} \\
& =\frac{c_{\Phi} n \omega_{n}^{1 / n}}{2}\left|E_{\lambda} \cap B_{r}\right|^{(n-1) / n}
\end{aligned}
$$

Furthermore, using $\lambda>C_{5}$, as in (4.26)

$$
\begin{aligned}
\int_{E_{\lambda} \cap B_{r}}|H| \mathrm{d} x & \leqslant \omega_{n}^{1 / n-1 / p} r^{1-n / p}\left(\int_{E_{\lambda} \cap B_{r_{1}}}|H|^{p} \mathrm{~d} x\right)^{1 / p}\left|E_{\lambda} \cap B_{r}\right|^{(n-1) / n} \\
& \leqslant \omega_{n}^{1 / n-1 / p}\left(C_{3} \lambda^{-1 / 2}\right)^{1-n / p}\left(\int_{E_{\lambda} \cap B_{r_{1}}}|H|^{p} \mathrm{~d} x\right)^{1 / p}\left|E_{\lambda} \cap B_{r}\right|^{(n-1) / n} \\
& \leqslant \frac{c_{\Phi} n \omega_{n}^{1 / n}}{2}\left|E_{\lambda} \cap B_{r}\right|^{(n-1) / n}
\end{aligned}
$$

Therefore, from (4.32) it follows that

$$
P_{\phi}\left(E_{\lambda}, B_{r}\right) \leqslant \int_{E_{\lambda} \cap \partial B_{r}} \phi\left(\nu_{B_{r}}\right) \mathrm{d} \mathcal{H}^{n-1}+c_{\Phi} n \omega_{n}^{1 / n}\left|E_{\lambda} \cap B_{r}\right|^{(n-1) / n} .
$$

Adding $\int_{E_{\lambda} \cap \partial B_{r}} \phi\left(\nu_{B_{r}}\right) \mathrm{d} \mathcal{H}^{n-1}$ to both sides of (4.33), using (2.5) and the isoperimetric inequality we get

$$
c_{\Phi} n \omega_{n}^{1 / n}\left|E_{\lambda} \cap B_{r}\right|^{(n-1) / n} \leqslant 4 C_{\Phi} \mathcal{H}^{n-1}\left(E_{\lambda} \cap \partial B_{r}\right) .
$$

Integrating this over $r$ we get the lower volume density estimate in (4.28).

To get the upper volume density estimate in (4.28) we use $\mathfrak{F}_{2}\left(E_{\lambda}, E_{0}, \lambda\right) \leqslant$ $\mathfrak{F}_{2}\left(E_{\lambda} \cup B_{r}, E_{0}, \lambda\right)$ and proceed as above.

For what concerns the upper perimeter density estimate in (4.29) we observe that from (4.33) and (2.5) it follows that

$$
2 c_{\Phi} P\left(E_{\lambda}, B_{r}\right) \leqslant\left(2 C_{\Phi}+c_{\Phi}\right) n \omega_{n} r^{n-1}
$$

for a.e. $r \in\left(0, C_{3} \lambda^{-1 / 2}\right)$. Since $r \mapsto P\left(E_{\lambda}, B_{r}\right)$ is nondecreasing and leftcontinuous, this inequality holds for all $r$. Finally the lower perimeter density estimate follows from (4.28) and the relative isoperimetric inequality for the ball. 
Step 5: Existence of GMM starting from $G$. We follow the arguments of $[42,48]$. Let $\{G(\lambda, k)\}_{\lambda>C_{5}, k \in \mathbb{N}_{0}}$ be defined as follows: $G(\lambda, 0)=G$ and

$$
G(\lambda, k) \in \operatorname{argmin} \mathfrak{F}_{2}(\cdot, G(\lambda, k-1), \lambda), \quad k \geqslant 1 .
$$

By step $1 G(\lambda, k)$ is well-defined and

$$
G(\lambda, k) \subseteq D
$$

for all $\lambda>C_{5}$ and $k \geqslant 0$. Notice also that

$$
k \in \mathbb{N}_{0} \mapsto P_{\phi}(G(\lambda, k))+\int_{G(\lambda, k)} H \mathrm{~d} x \quad \text { is nonincreasing. }
$$

Given $t>s>0$ with $t-s<1$, let $\lambda>\max \left\{C_{5}, 5+C_{3}^{-2} / t-s, 5 / s\right\}$ so that $[\lambda t]-[\lambda s] \geqslant 4, \quad[\lambda s] \geqslant 5$ and $1 / \lambda|t-s|^{1 / 2}<C_{3} \lambda^{-1 / 2}$. By proposition 3.3 applied with $A=G(\lambda, k-1), \quad r_{0}=C_{3} \lambda^{-1 / 2}, \quad \theta:=C_{4}, \quad \ell:=1 / \lambda|t-s|^{1 / 2} \quad$ and $B=$ $G(\lambda, k)$, and using the bounds (2.5) and (4.1) for anisotropies and mobilities, for any $k \in\{[\lambda s]+1, \ldots,[\lambda t]\}$ we get

$$
\begin{aligned}
|G(\lambda, k-1) \Delta G(\lambda, k)| \leqslant & \frac{5^{n} \omega_{n}}{2 C_{4} c_{\Phi} \lambda|t-s|^{1 / 2}} P_{\phi}(G(\lambda, k-1)) \\
& +\frac{\lambda|t-s|^{1 / 2}}{2 c_{\Psi}} \int_{G(\lambda, k-1) \Delta G(\lambda, k)} d_{\psi}^{G(\lambda, k-1)} \mathrm{d} x .
\end{aligned}
$$

Therefore,

$$
\begin{aligned}
|G(\lambda,[\lambda s]) \Delta G(\lambda,[\lambda t])| \leqslant & \sum_{k=[\lambda s]+1}^{[\lambda t]}|G(\lambda, k-1) \Delta G(\lambda, k)| \\
\leqslant & \frac{5^{n} \omega_{n}}{2 C_{4} c_{\Phi} \lambda|t-s|^{1 / 2}} \sum_{k=[\lambda s]+1}^{[\lambda t]} P_{\phi}(G(\lambda, k-1)) \\
& +\frac{\lambda|t-s|^{1 / 2}}{2 c_{\Psi}} \sum_{k=[\lambda s]+1}^{[\lambda t]} \int_{G(\lambda, k-1) \Delta G(\lambda, k)} d_{\psi}^{G(\lambda, k-1)} \mathrm{d} x
\end{aligned}
$$

By (4.35),

$$
\begin{aligned}
& \sum_{k=[\lambda s]+1}^{[\lambda t]} P_{\phi}(G(\lambda, k-1)) \\
& \leqslant \sum_{k=[\lambda s]+1}^{[\lambda t]}\left(P_{\phi}(G(\lambda, k-1))+\int_{G(\lambda, k-1)} H \mathrm{~d} x+\int_{G(\lambda, k-1)}|H| \mathrm{d} x\right) \\
& \leqslant\left(P_{\phi}(G)+\int_{G} H \mathrm{~d} x+\int_{D}|H| \mathrm{d} x\right)([\lambda t]-[\lambda s]) \\
& \leqslant\left(P_{\phi}(G)+2 \int_{D}|H| \mathrm{d} x\right)(\lambda(t-s)+1)
\end{aligned}
$$


and

$$
\begin{aligned}
& \lambda \sum_{k=[\lambda s]+1}^{[\lambda t]} \int_{G(\lambda, k-1) \Delta G(\lambda, k)} d_{\psi}^{G(\lambda, k-1)} \mathrm{d} x \\
& \quad \leqslant P_{\phi}(G(\lambda,[\lambda s]))+\int_{G(\lambda,[\lambda s])} H \mathrm{~d} x-P_{\phi}(G(\lambda,[\lambda t]))-\int_{G(\lambda,[\lambda t])} H \mathrm{~d} x \\
& \quad \leqslant P_{\phi}(G)+2 \int_{D}|H| \mathrm{d} x
\end{aligned}
$$

therefore, from (4.36) we get

$$
|G(\lambda,[\lambda s]) \Delta G(\lambda,[\lambda t])| \leqslant\left(C_{6}|t-s|^{1 / 2}+\frac{C_{6}-1 / 2 c_{\Psi}}{\lambda|t-s|^{1 / 2}}\right)\left(P_{\phi}(G)+2 \int_{D}|H| \mathrm{d} x\right)
$$

where

$$
C_{6}:=\frac{5^{n} \omega_{n}}{2 C_{4} c_{\Phi}}+\frac{1}{2 c_{\Psi}} .
$$

Now (4.18) and (4.19) follow from (4.37) and (4.34), respectively.

We will use (4.29), (4.30) and (4.37) in the proof of theorem 5.1.

\section{Improved time Hölder regularity}

In this section we show that when $\phi_{i}=\phi$ and $\psi_{i}=\psi$ for any $i=1, \ldots, N+1$, the time Hölder continuity exponent of GMM for partitions can be improved to $1 / 2$. The result follows from the generalization of $[42]$ in the previous section (theorem 4.2) combined with a comparison (theorem 5.2 below) between a multiphase flow and a two-phase flow starting from just one of the phases and its complement. Arguments from our main continuity result (in theorem 4.1) are needed to reconnect both flows in the limit.

Theorem 5.1. Let $\Phi=\{\phi, \ldots, \phi\}$ and $\Psi=\{\psi, \ldots, \psi\}$ for some norms $\phi$ and $\psi$ on $\mathbb{R}^{n}$, and $\boldsymbol{H} \equiv 0$. Then for any $\mathcal{G} \in \mathbb{P}_{b}(N+1)$ and $\mathcal{M} \in \operatorname{GMM}(\mathfrak{F}, \mathcal{G})$

$$
\left|\mathcal{M}(t) \Delta \mathcal{M}\left(t^{\prime}\right)\right| \leqslant C_{6} \operatorname{Per}_{\Phi}(\mathcal{G})\left|t-t^{\prime}\right|^{1 / 2}, \quad t, t^{\prime}>0,\left|t-t^{\prime}\right|<1,
$$

where $C_{6}$ is given in (4.38). In addition, if $\sum_{j=1}^{N+1}\left|\overline{G_{j}} \backslash G_{j}\right|=0$, then (5.1) holds for any $t, t^{\prime} \geqslant 0$ with $\left|t-t^{\prime}\right|<1$.

Recall that, by theorem 4.1, for any $\mathcal{G} \in \mathbb{P}_{b}(N+1), G M M(\mathfrak{F}, \mathcal{G})$ is nonempty, each $\mathcal{M} \in G M M(\mathfrak{F}, \mathcal{G})$ is locally $1 /(n+1)$-Hölder continuous and

$$
\bigcup_{i=1}^{N} M_{i}(t) \subseteq \operatorname{co}(\mathcal{G}) \text { for any } t \geqslant 0
$$


Besides $\mathfrak{F}$ we need to consider also the functional $\mathfrak{F}_{2}$ defined (up to constants) in (4.17) with $H=0$, i.e.,

$$
\mathfrak{F}_{2}(G, E, \lambda):=P_{\phi}(G)+\lambda \int_{G \Delta E} d_{\psi}(x, \partial E) \mathrm{d} x .
$$

We start with a comparison result: this is the key point of the proof of theorem 5.1 since it allows to compare the evolution of a single phase with the multiphase case.

THEOREM 5.2 Discrete comparison multiphase-phase. Let $g_{1}, \ldots, g_{N+1} \in L_{\text {loc }}^{1}\left(\mathbb{R}^{n}\right)$ and suppose that $\mathcal{A} \in \mathbb{P}_{b}(N+1)$ minimize

$$
\mathcal{E} \in \mathbb{P}_{b}(N+1) \mapsto \sum_{i=1}^{N+1} P_{\phi}\left(E_{i}\right)+\sum_{i=1}^{N} \int_{E_{i}} g_{i} \mathrm{~d} x-\int_{E_{N+1}^{c}} g_{N+1} \mathrm{~d} x
$$

Suppose that for $i \in\{1, \ldots, N\}$ and $g_{i}^{\prime} \in L_{\text {loc }}^{1}\left(\mathbb{R}^{n}\right)$, there exists a bounded minimizer $F_{i}$ of

$$
F \in B V\left(\mathbb{R}^{n} ;\{0,1\}\right) \mapsto P_{\phi}(F)+\int_{F} g_{i}^{\prime} \mathrm{d} x
$$

and suppose that, given $g_{N+1}^{\prime} \in L_{\mathrm{loc}}^{1}\left(\mathbb{R}^{n}\right)$, there exists a bounded minimizer of

$$
G \in B V\left(\mathbb{R}^{n} ;\{0,1\}\right) \mapsto P_{\phi}(G)-\int_{G} g_{N+1}^{\prime} \mathrm{d} x
$$

the complement of which we denote by $F_{N+1}$. If $2 g_{i}^{\prime}-g_{i}+g_{j}>0$ a.e. in $\mathbb{R}^{n}$ for all $i, j \in\{1, \ldots, N+1\}, i \neq j$, then

$$
F_{i} \subseteq A_{i}, \quad i \in\{1, \ldots, N+1\}
$$

Proof. Let $i \in\{1, \ldots, N\}$. By minimality,

$$
\begin{aligned}
& \sum_{j=1}^{N+1} P_{\phi}\left(A_{j}\right)+\sum_{j=1}^{N} \int_{A_{j}} g_{j} \mathrm{~d} x-\int_{A_{N+1}^{c}} g_{N+1} \mathrm{~d} x \leqslant P_{\phi}\left(A_{i} \cup F_{i}\right) \\
& \quad+\sum_{j=1, j \neq i}^{N+1} P_{\phi}\left(A_{j} \backslash F_{i}\right)+\int_{A_{i} \cup F_{i}} g_{i} \mathrm{~d} x+\sum_{j=1, j \neq i}^{N} \int_{A_{j} \backslash F_{i}} g_{j} \mathrm{~d} x-\int_{A_{N+1}^{c} \cup F_{i}} g_{N+1} \mathrm{~d} x
\end{aligned}
$$

and

$$
P_{\phi}\left(F_{i}\right)+\int_{F_{i}} g_{i}^{\prime} \mathrm{d} x \leqslant P_{\phi}\left(F_{i} \cap A_{i}\right)+\int_{F_{i} \cap A_{i}} g_{i}^{\prime} \mathrm{d} x
$$


Summing (5.3) and twice (5.4), we obtain

$$
\begin{aligned}
& P_{\phi}\left(F_{i}\right)+P_{\phi}\left(A_{i}\right)+P_{\phi}\left(F_{i}\right)+\sum_{j=1, j \neq i}^{N+1} P_{\phi}\left(A_{j}\right) \\
& \quad+\int_{F_{i} \backslash A_{i}}\left(g_{i}^{\prime}-g_{i}\right) \mathrm{d} x+\sum_{j=1, j \neq i}^{N+1} \int_{A_{j} \cap F_{i}} g_{j} \mathrm{~d} x+\int_{F_{i} \backslash A_{i}} g_{i}^{\prime} \mathrm{d} x \\
& \leqslant P_{\phi}\left(F_{i} \cup A_{i}\right)+P_{\phi}\left(F_{i} \cap A_{i}\right)+\sum_{j=1, j \neq i}^{N+1} P_{\phi}\left(A_{j} \backslash F_{i}\right)+P_{\phi}\left(F_{i} \cap A_{i}\right) .
\end{aligned}
$$

Let us show that for any $E \in B V\left(\mathbb{R}^{n} ;\{0,1\}\right), \mathcal{G} \in \mathbb{P}_{b}(N+1)$ and $i \in\{1, \ldots$, $N+1\}$,

$$
\sum_{j=1, j \neq i}^{N+1} P_{\phi}\left(G_{j} \backslash E\right)+P_{\phi}\left(G_{i} \cap E\right) \leqslant P_{\phi}(E)+\sum_{j=1, j \neq i}^{N+1} P_{\phi}\left(G_{j}\right) .
$$

First assume that $\mathcal{H}^{n-1}\left(\partial^{*} E \cap \bigcup_{j=1}^{N+1} \partial^{*} G_{j}\right)=0$. In this case by $(2.1)$ and $(2.2)$, as well as the inclusion $\partial^{*} G_{i} \subset \bigcup_{j=1, j \neq i}^{N+1} \partial^{*} G_{j}$, we obtain

$$
P_{\phi}\left(G_{j} \backslash E\right)=\int_{E^{(0)} \cap \partial^{*} G_{j}} \phi\left(\nu_{G_{j}}\right) \mathrm{d} \mathcal{H}^{n-1}+\int_{G_{j} \cap \partial^{*} E} \phi\left(\nu_{E}\right) \mathrm{d} \mathcal{H}^{n-1}
$$

and

$$
\begin{aligned}
P_{\phi}\left(G_{i} \cap E\right) & =\int_{E \cap \partial^{*} G_{i}} \phi\left(\nu_{G_{i}}\right) \mathrm{d} \mathcal{H}^{n-1}+\int_{G_{i} \cap \partial^{*} E} \phi\left(\nu_{E}\right) \mathrm{d} \mathcal{H}^{n-1} \\
& =\sum_{j=1, j \neq i}^{N+1} \int_{E \cap \partial^{*} G_{j} \cap \partial^{*} G_{i}} \phi\left(\nu_{G_{j}}\right) \mathrm{d} \mathcal{H}^{n-1}+\int_{G_{i} \cap \partial^{*} E} \phi\left(\nu_{E}\right) \mathrm{d} \mathcal{H}^{n-1},
\end{aligned}
$$

and hence,

$$
\begin{aligned}
& \sum_{j=1, j \neq i}^{N+1} P_{\phi}\left(G_{j} \backslash E\right)+P_{\phi}\left(G_{i} \cap E\right) \\
& =\sum_{j=1, j \neq i}^{N+1}\left(\int_{E^{(0)} \cap \partial^{*} G_{j}} \phi\left(\nu_{G_{j}}\right) \mathrm{d} \mathcal{H}^{n-1}+\int_{E \cap \partial^{*} G_{j} \cap \partial^{*} G_{i}} \phi\left(\nu_{G_{j}}\right) \mathrm{d} \mathcal{H}^{n-1}\right) \\
& \quad+\sum_{j=1}^{N+1} \int_{G_{j} \cap \partial^{*} E} \phi\left(\nu_{E}\right) \mathrm{d} \mathcal{H}^{n-1} \leqslant \sum_{j=1, j \neq i}^{N+1} P_{\phi}\left(G_{j}\right)+P_{\phi}(E) .
\end{aligned}
$$

In the general case we choose a sequence $\left\{\xi_{k}\right\} \subset \mathbb{R}^{n}$ such that $\left|\xi_{k}\right| \rightarrow 0$ and $\mathcal{H}^{n-1}\left(\partial^{*}\left(E+\xi_{k}\right) \cap \bigcup_{j=1}^{N+1} \partial^{*} G_{j}\right)=0$, where $E+\xi_{k}:=\left\{x \in \mathbb{R}^{n}: x-\xi_{k} \in\right.$ 
$E\}$. By the previous case,

$$
\sum_{j=1, \neq i}^{N+1} P_{\phi}\left(G_{j} \backslash\left(E+\xi_{k}\right)\right)+P_{\phi}\left(G_{i} \cap\left(E+\xi_{k}\right)\right) \leqslant \sum_{j=1, j \neq i}^{N+1} P_{\phi}\left(G_{j}\right)+P_{\phi}\left(E+\xi_{k}\right) .
$$

Since $P_{\phi}\left(E+\xi_{k}\right)=P_{\phi}(E)$ and $\lim _{k \rightarrow+\infty}\left|\left(E+\xi_{k}\right) \Delta E\right| \rightarrow 0$, letting $k \rightarrow+\infty$ in (5.7) and using the $L^{1}\left(\mathbb{R}^{n}\right)$-lower semicontinuity of the $\phi$-perimeter we get (5.6). Inserting (5.6) with $\mathcal{G}=\mathcal{A}$ and $E=F_{i}$ in (5.5) and using (2.4) we get

$$
\int_{F_{i} \backslash A_{i}}\left(g_{i}^{\prime}-g_{i}\right) \mathrm{d} x+\sum_{j=1, j \neq i}^{N+1} \int_{A_{j} \cap F_{i}} g_{j} \mathrm{~d} x+\int_{F_{i} \backslash A_{i}} g_{i}^{\prime} \mathrm{d} x \leqslant 0 .
$$

Recall that $F_{i} \backslash A_{i}=\bigcup_{j=1, j \neq i}^{N+1} F_{i} \cap A_{j}$ up to a negligible set, thus,

$$
\sum_{j=1, j \neq i}^{N+1} \int_{A_{j} \cap F_{i}}\left(2 g_{i}^{\prime}-g_{i}+g_{j}\right) \mathrm{d} x \leqslant 0 .
$$

By assumption $2 g_{i}^{\prime}-g_{i}+g_{j}>0$ a.e., and hence $F_{i} \subseteq A_{i}$ up to a negligible set. The case $i=N+1$ is similar.

Lemma 5.3. Let $\mathcal{G} \in \mathbb{P}_{b}(N+1)$ and set

$$
g_{j}(\cdot):=\widetilde{d}_{\psi}\left(\cdot, \partial G_{j}\right), \quad j \in\{1, \ldots, N+1\} .
$$

For $E \subseteq \mathbb{R}^{n}$ define $e(\cdot)=\widetilde{d}_{\psi}(\cdot, \partial E)$. If either $E \subseteq G_{i}$ for some $i \in\{1, \ldots, N\}$ or $G_{N+1}^{c} \subseteq E^{c}$, then $2 e-g_{i}+g_{j} \geqslant 0$ a.e. in $\mathbb{R}^{n}$ for any $j \in\{1, \ldots, N+1\}, j \neq$ i. Similarly, if either $E \subset \subset G_{i}$ for some $i \in\{1, \ldots, N\}$ or $G_{N+1}^{c} \subset \subset E^{c}$, then $2 e-g_{i}+g_{j} \geqslant 0$ a.e. in $\mathbb{R}^{n}$ for any $j \in\{1, \ldots, N+1\}, j \neq i$.

Proof. Since $E_{i}^{c} \subseteq G_{i}^{c} \cup G_{j}^{c}$, the assertion follows from the relation

$$
A \subseteq B \Longrightarrow \widetilde{d}_{\psi}(\cdot, \partial A) \geqslant \widetilde{d}_{\psi}(\cdot, \partial B) \text { a.e. in } \mathbb{R}^{n}
$$

Lemma 5.4. Given $\mathcal{A} \in \mathbb{P}_{b}(N+1)$, let $\mathcal{A}(\lambda)$ minimize $\mathfrak{F}(\cdot, \mathcal{A}, \lambda)$ with $\boldsymbol{H}=0$. For $i \in\{1, \ldots, N+1\}$ let $E \in B V\left(\mathbb{R}^{n} ;\{0,1\}\right)$ be such that $E \subseteq A_{i}$; in case $i=$ $N+1$ we assume also that $E^{c}$ is bounded. Then there exists a minimizer $E_{i}(\lambda)$ of $\mathfrak{F}_{2}(\cdot, E, \lambda)$ such that $E_{i}(\lambda) \subseteq A_{i}(\lambda)$.

Proof. First we assume that $E=A_{i}$. Let $E_{1} \subset \subset E_{2} \subset \subset \ldots \subset \subset A_{i}$ be sets of finite perimeter such that $A_{i}=\bigcup_{k} E_{k}$ and $\widetilde{d}\left(\cdot, \partial E_{k}\right) \rightarrow \widetilde{d}\left(\cdot, \partial A_{i}\right)$ a.e. as $k \rightarrow+\infty$. Let $E_{k}(\lambda)$ be a minimizer of $\mathfrak{F}_{2}\left(\cdot, E_{k}, \lambda\right)$. By $[\mathbf{1 8}], E_{1}(\lambda) \subseteq E_{2}(\lambda) \ldots$ and $E(\lambda)_{*}:=$ $\bigcup_{k} E_{k}(\lambda)$ is the minimal minimizer of $\mathfrak{F}_{2}\left(\cdot, A_{i}, \lambda\right)$. Since $E_{k} \subset \subset A_{i}$, by lemma 5.3,

$$
2 \widetilde{d}\left(\cdot, \partial E_{k}\right)-\widetilde{d}\left(\cdot, \partial A_{i}\right)+\widetilde{d}\left(\cdot, \partial A_{j}\right)>0 \text { a.e. in } \mathbb{R}^{n} \text { for all } j \neq i \text {. }
$$

Thus, by theorem 5.2, $E_{k}(\lambda) \subseteq A_{i}(\lambda)$. Hence, we get $E(\lambda)_{*} \subseteq A_{i}(\lambda)$. 
In the general case, we consider the minimal minimizer $E(\lambda)_{*}$ of $\mathfrak{F}_{2}(\cdot, E, \lambda)$ and the minimal minimizer $A_{i}(\lambda)_{*}$ of $\mathfrak{F}_{2}\left(\cdot, A_{i}, \lambda\right)$. Since $E \subseteq A_{i}$, by $[\mathbf{1 8}], E(\lambda)_{*} \subseteq$ $A_{i}(\lambda)_{*}$. Hence, $E_{i}(\lambda)=E(\lambda)_{*}$ satisfies the assertion of the lemma.

Proof of theorem 5.1. Given $\mathcal{G} \in \mathbb{P}_{b}(N+1)$ define $\{\mathcal{G}(\lambda, k)\}_{\lambda \geqslant 1, k \in \mathbb{N}_{0}}$ as follows: $\mathcal{G}(\lambda, 0)=\mathcal{G}$ and

$$
\mathcal{G}(\lambda, k) \in \operatorname{argmin} \mathfrak{F}(\cdot, \mathcal{G}(\lambda, k-1), \lambda), \quad k \geqslant 1 .
$$

Note that the map $k \in \mathbb{N}_{0} \mapsto \operatorname{Per}_{\Phi}(\mathcal{G}(\lambda, k))$ is nonincreasing. In particular,

$$
\operatorname{Per}_{\Phi}(\mathcal{G}(\lambda, k)) \leqslant \operatorname{Per}_{\Phi}(\mathcal{G})
$$

For any $i \in\{1, \ldots, N+1\}$ and $k \geqslant 0$, let $\left\{F_{i}^{k}(\lambda, l)\right\}_{l \geqslant k}$ be defined as follows: $F_{i}^{k}(\lambda, k):=G_{i}(\lambda, k)$ and $F_{i}^{k}(\lambda, l)$ is the minimal minimizer of $\mathfrak{F}_{2}\left(\cdot, F_{i}^{k}(\lambda, l-1), \lambda\right)$ for $l>k$. Notice that, according to step 2 of the proof of theorem 4.1, our actual initial set $F_{i}^{k}(\lambda, k)=G_{i}(\lambda, k)$ satisfies the density estimates (4.9) and (4.10) for all radii $r \leqslant O(1 / \lambda)$ and, according to the proof of step 4 of theorem 4.2 , all $F_{i}^{k}(\lambda, l)$, $l>k$, satisfy the density estimates (4.28) and (4.29) for all radii $r \leqslant O\left(1 / \lambda^{1 / 2}\right)$. Moreover, since the initial set $F_{i}^{k}(\lambda, k)$ also depends on $\lambda$, we cannot use the arguments of the $1 /(n+1)$-Hölder continuity up to time 0 in the proof of theorem 4.1 .

For shortness we call $\left\{F_{i}^{k}(\lambda, l)\right\}_{l \geqslant k}$ a discrete solution starting from $F_{i}^{k}(\lambda, k)=$ $G_{i}(\lambda, k)$. Applying lemma 5.4 inductively one can show that

$$
F_{i}^{k}(\lambda, l) \subseteq G_{i}(\lambda, l), \quad l \geqslant k .
$$

In particular,

$$
G_{i}(\lambda, l)=\left(\bigcup_{j \neq i} G_{j}(\lambda, l)\right)^{c}=\bigcap_{j \neq i} G_{j}(\lambda, l)^{c} \subseteq \bigcap_{j \neq i} F_{j}^{k}(\lambda, l)^{c} .
$$

Hence, using $F_{i}^{k}(\lambda, k):=G_{i}(\lambda, k)$ for all $i=1, \ldots, N+1$, we get

$$
G_{i}(\lambda, l) \backslash G_{i}(\lambda, k) \subseteq\left(\bigcap_{j \neq i} F_{j}^{k}(\lambda, l)^{c}\right) \cap\left(\bigcup_{j \neq i} F_{j}^{k}(\lambda, l)\right) \subseteq \bigcup_{j \neq i}\left(F_{j}^{k}(\lambda, k) \backslash F_{j}^{k}(\lambda, l)\right) .
$$

On the other hand,

$$
G_{i}(\lambda, k) \backslash G_{i}(\lambda, l)=F_{i}^{k}(\lambda, k) \backslash G_{i}(\lambda, l) \subseteq F_{i}^{k}(\lambda, k) \backslash F_{i}^{k}(\lambda, l),
$$

hence,

$$
|\mathcal{G}(\lambda, k) \Delta \mathcal{G}(\lambda, l)| \leqslant \sum_{i=1}^{N+1}\left|F_{i}^{k}(\lambda, k) \backslash F_{i}^{k}(\lambda, l)\right|,
$$

which is the inequality that will allow us to get the $1 / 2$-Hölderianity of GMM. 
Fix $i \in\{1, \ldots, N+1\}$ and choose arbitrary $t>s^{\prime}>s>0$. Let $\left\{F_{i}^{[\lambda s]}(\lambda, l)\right\}_{l \geqslant[\lambda s]}$ be a discrete solution starting from $F_{i}^{[\lambda s]}(\lambda,[\lambda s])=G_{i}(\lambda,[\lambda s])$. Then for any $\lambda>\left(5 / s^{\prime}-s\right)+\left(5 / t-s^{\prime}\right)+(5 / s)$ we have

$$
\begin{aligned}
\left.\mid F_{i}^{[\lambda s]}(\lambda,[\lambda s])\right) \backslash F_{i}^{[\lambda s]}(\lambda,[\lambda t]) \mid \leqslant & \sum_{l=[\lambda s]+1}^{\left[\lambda s^{\prime}\right]}\left|F_{i}^{[\lambda s]}(\lambda, l) \Delta F_{i}^{[\lambda s]}(\lambda, l-1)\right| \\
& +\sum_{l=\left[\lambda s^{\prime}\right]+1}^{[\lambda t]}\left|F_{i}^{[\lambda s]}(\lambda, l) \Delta F_{i}^{[\lambda s]}(\lambda, l-1)\right|=: I_{1}+I_{2} .
\end{aligned}
$$

Note that by the choice of $\lambda$, we have $\left[\lambda s^{\prime}\right]-[\lambda s] \geqslant 4,[\lambda t]-\left[\lambda s^{\prime}\right] \geqslant 4$ and $[\lambda s] \geqslant$ 4. According to step 4 of the proof of theorem $4.2, F_{i}^{[\lambda s]}(\lambda, l), l \geqslant[\lambda s] \geqslant 4$ satisfies the uniform lower perimeter density estimate

$$
C_{4} \leqslant \frac{P\left(F_{i}^{[\lambda s]}(\lambda, l), B_{r}(x)\right)}{r^{n-1}}, \quad x \in \partial F_{i}^{[\lambda s]}(\lambda, l), \quad r \in\left(0, C_{3} \lambda^{-1 / 2}\right),
$$

provided $\lambda>C_{5}$. Hence, from (4.37),

$$
I_{2} \leqslant\left(C_{6}\left|t-s^{\prime}\right|^{1 / 2}+\frac{C_{6}-1 / 2 c_{\Psi}}{\lambda\left|t-s^{\prime}\right|^{1 / 2}}\right) P_{\phi}\left(G_{i}(\lambda,[\lambda s])\right) .
$$

Since $\mathcal{G}(\lambda,[\lambda s])$ minimizes $\mathfrak{F}(\cdot, \mathcal{G}(\lambda,[\lambda s]-1), \lambda)$, by step 3 of the proof of theorem 4.1 , see in particular $(4.8)$ and $(4.10)$,

$$
c^{\Phi}(N, n) \leqslant \frac{P\left(G_{i}(\lambda,[\lambda s]), B_{r}(x)\right)}{r^{n-1}}, \quad x \in \partial G_{i}(\lambda,[\lambda s]), \quad r \in\left(0, \frac{C(n, N, p, \Phi, \Psi)}{\lambda}\right) .
$$

Because of the presence of $1 / \lambda$ (instead of $\left.1 / \lambda^{1 / 2}\right)$ in $(5.11)$, in general we cannot use (4.37). To estimate $I_{1}$ we proceed as in the proof of (4.15) and get

$$
I_{1} \leqslant\left(\mathrm{C}\left|s^{\prime}-s\right|^{1 / n+1}+\frac{\widetilde{\mathrm{C}}}{\lambda\left|s^{\prime}-s\right|^{n / n+1}}\right) P_{\phi}\left(G_{i}(\lambda,[\lambda s])\right) .
$$

From the estimates for $I_{1}$ and $I_{2}$, and (5.8), (5.9) and (5.10) we obtain

$$
\begin{aligned}
|\mathcal{G}(\lambda,[\lambda s]) \Delta \mathcal{G}(\lambda,[\lambda t])| & \\
\leqslant & \left(\mathrm{C}\left|s^{\prime}-s\right|^{1 / n+1}+\frac{\widetilde{\mathrm{C}}}{\lambda\left|s^{\prime}-s\right|^{n / n+1}}\right) \operatorname{Per}_{\Phi}(\mathcal{G}(\lambda,[\lambda s])) \\
& +\left(C_{6}\left|t-s^{\prime}\right|^{1 / 2}+\frac{C_{6}-1 / 2 c_{\Psi}}{\lambda\left|t-s^{\prime}\right|^{1 / 2}}\right) \operatorname{Per}_{\Phi}(\mathcal{G}(\lambda,[\lambda s])) \\
\leqslant & \left(C\left|s^{\prime}-s\right|^{1 / n+1}+\frac{\widetilde{\mathrm{C}}}{\lambda\left|s^{\prime}-s\right|^{n / n+1}}+C_{6}\left|t-s^{\prime}\right|^{1 / 2}+\frac{C_{6}-1 / 2 c_{\Psi}}{\lambda\left|t-s^{\prime}\right|^{1 / 2}}\right) \operatorname{Per}_{\Phi}(\mathcal{G}) .
\end{aligned}
$$


Now if $\mathcal{M} \in G M M(\mathfrak{F}, \mathcal{G})$, there exists $\lambda_{k} \rightarrow+\infty$ for which

$$
\lim _{k \rightarrow \infty}\left|\mathcal{G}\left(\lambda_{k},\left[\lambda_{k} t\right]\right) \Delta \mathcal{M}(t)\right|=0 \text { for any } t \geqslant 0 .
$$

Thus, from (5.12) we get

$$
|\mathcal{M}(s) \Delta \mathcal{M}(t)| \leqslant\left(\mathrm{C}\left|s^{\prime}-s\right|^{1 / n+1}+C_{6}\left|t-s^{\prime}\right|^{1 / 2}\right) \operatorname{Per}_{\Phi}(\mathcal{G})
$$

Since $s^{\prime} \in(s, t)$ is arbitrary, letting $s^{\prime} \searrow s$ we get (5.1) with $C:=C_{6}$.

Finally, if $\sum_{j=1}^{N+1}\left|\overline{G_{j}} \backslash G_{j}\right|=0$, then for any $t>s>0$ and $\mathcal{M} \in \operatorname{GMM}(\mathfrak{F}, \mathcal{G})$ we have

$$
|\mathcal{M}(t) \Delta \mathcal{G}| \leqslant|\mathcal{M}(t) \Delta \mathcal{M}(s)|+|\mathcal{M}(s) \Delta \mathcal{G}| \leqslant C_{6} \operatorname{Per}_{\Phi}(\mathcal{G})|t-s|^{1 / 2}+\mathrm{Cs}^{1 / n+1},
$$

where in the second inequality we used (5.1) and (4.5). Now letting $s \searrow 0$ we get

$$
|\mathcal{M}(t) \Delta \mathcal{G}| \leqslant C_{6} \operatorname{Per}_{\Phi}(\mathcal{G}) t^{1 / 2} .
$$

From lemma 5.4 we get the following weak comparison property of GMM.

Theorem 5.5 Comparison. Let $\Phi=\{\phi, \ldots, \phi\}$ and $\Psi=\{\psi, \ldots, \psi\}$ for some norms $\phi$ and $\psi$ on $\mathbb{R}^{n}$, and $\boldsymbol{H} \equiv 0$. Given $\mathcal{G} \in \mathbb{P}_{b}(N+1)$, let $\mathcal{M} \in G M M(\mathfrak{F}, \mathcal{G})$ and given $i \in\{1, \ldots, N+1\}$, let $C \in B V\left(\mathbb{R}^{n} ;\{0,1\}\right)$ be such that $C \subseteq G_{i}$; in case $i=N+1$ we assume also that $C^{c}$ is bounded. Then there exists $N \in$ $G M M\left(\mathfrak{F}_{2}, C\right)$ such that $N(t) \subseteq M_{i}(t)$ for all $t \geqslant 0$.

Proof. Let $\lambda_{h} \rightarrow+\infty$ be such that

$$
\lim _{h \rightarrow \infty}\left|\mathcal{G}\left(\lambda_{h},\left[\lambda_{h} t\right]\right) \Delta \mathcal{M}(t)\right|=0 \quad \text { for all } t \geqslant 0
$$

where for any $h$ the sequence $\left\{\mathcal{G}\left(\lambda_{h}, k\right)\right\}_{k \in \mathbb{N}_{0}}$ is defined as: $\mathcal{G}\left(\lambda_{h}, 0\right)=\mathcal{G}$ and

$$
\mathcal{G}\left(\lambda_{h}, k\right) \in \operatorname{argmin} \mathfrak{F}\left(\cdot, \mathcal{G}\left(\lambda_{h}, k-1\right), \lambda_{h}\right), \quad k \geqslant 1 .
$$

Let $i \in\{1, \ldots, N+1\}$ and $C \in B V\left(\mathbb{R}^{n} ;\{0,1\}\right)$ be as in the statement. For any $h$ let $\left\{G\left(\lambda_{h}, k\right)\right\}_{k \in \mathbb{N}_{0}}$ be defined as $G\left(\lambda_{h}, 0\right)=C$ and $G\left(\lambda_{h}, k\right)$ is the minimal minimizer of $\mathfrak{F}_{2}\left(\cdot, G\left(\lambda_{h}, k-1\right), \lambda\right), \quad k \geqslant 1$ (see the proof of lemma 5.4 for the definition). Applying lemma 5.4 inductively we get

$$
G\left(\lambda_{h}, k\right) \subseteq G_{i}\left(\lambda_{h}, k\right) \text { for all } h \geqslant 1 \text { and } k \geqslant 0 .
$$

Passing to a further (not relabelled) subsequence if necessary, we assume that there exists $N \in G M M\left(\mathfrak{F}_{2}, C\right)$ such that

$$
\lim _{h \rightarrow \infty}\left|G\left(\lambda_{h},\left[\lambda_{h} t\right]\right) \Delta N(t)\right|=0 \text { for all } t \geqslant 0 .
$$

By (5.13) we have

$$
\lim _{h \rightarrow \infty}\left|G_{i}\left(\lambda_{h},\left[\lambda_{h} t\right]\right) \Delta M_{i}(t)\right|=0 \quad \text { for all } t \geqslant 0 .
$$

Now (5.14) and (5.15) imply that $N(t) \subseteq M_{i}(t)$ for all $t \geqslant 0$ up to a negligible set. 
Corollary 5.6. Under the assumptions of theorem 5.5 let $\mathcal{G} \in \mathbb{P}_{b}(N+1)$ and $\mathcal{M} \in G M M(\mathfrak{F}, \mathcal{G})$. Let $C_{i} \subseteq G_{i}, \quad i \in\{1, \ldots, N\}$, and $C_{N+1}^{c} \supseteq \operatorname{co}(\mathcal{G})$ be convex sets and let $L_{i} \in G M M\left(\mathfrak{F}_{2}, C_{i}\right), \quad i \in\{1, \ldots, N+1\}$. Then for any $\mathcal{M} \in$ $\operatorname{GMM}(\mathfrak{F}, \mathcal{G})$

$$
L_{i}(t) \neq \emptyset \quad \Longrightarrow \quad M_{i}(t) \neq \emptyset, \quad i=1, \ldots, N
$$

and

$$
L_{N+1}(t)=\emptyset \quad \Longrightarrow \quad M_{N+1}(t)=\emptyset .
$$

Proof. Recall that anisotropic mean curvature flow with a mobility starting from a bounded convex set $C$ is uniquely defined [10], coincides with the GMM starting from $C$ and becomes extinct at a finite time $t_{C}>0$. By theorem 5.5, the $i$-th phase $M_{i}$ of any $\mathcal{M} \in G M M(\mathfrak{F}, \mathcal{G})$ starting from the $i$-th phase $G_{i}$ of $\mathcal{G}$ does not disappear in the time-interval $\left(0, t_{C_{i}}\right)$ for any $i \in\{1, \ldots, N\}$. Analogously, theorem 5.5 implies that $(N+1)$-th phase of $\mathcal{M}$ becomes empty, i.e., $\mathbb{R}^{n} \backslash M_{N+1}(t)=\emptyset$ if $t \geqslant t_{C_{N+1}}$.

\section{Acknowledgements}

The first author acknowledges support from GNAMPA (Gruppo Nazionale per l'Analisi Matematica, la Probabilità e le loro Applicazioni) of INdAM (Istituto Nazionale di Alta Matematica). The third author acknowledges support from the Austrian Science Fund (FWF) projects M 2571-N32 and P 29681.

\section{References}

1 F. Almgren, J. Taylor and L. Wang. Curvature-driven flows: a variational approach. SIAM J. Control Optim. 31 (1993), 387-438. (doi: 10.1137/0331020).

2 F. Almgren and J. E. Taylor. Flat flow is motion by crystalline curvature for curves with crystalline energies. J. Differential Geom. 42 (1995), 1-22. (doi:10.4310/jdg/1214457030).

3 L. Ambrosio. Movimenti minimizzanti. Rend. Accad. Naz. Sci. XL Mem. Mat. Appl. 19 (1995), 191-246. (MR1387558).

4 L. Ambrosio, N. Fusco and D. Pallara. Functions of bounded variation and free biscontinuity problems (New York: Oxford University Press, 2000).

5 S. B. Angenent and M. E. Gurtin. Multiphase thermomechanics with interfacial structure 2. Evolution of an isothermal interface. Arch. Rational Mech. Anal. 108 (1989), 323-391. (doi: 10.1007/BF01041068).

6 G. Bellettini, M. Novaga and M. Paolini. On a crystalline variational problem, Part I: first variation and global $L^{\infty}$ regularity. Arch. Rational Mech. Anal. 157 (2001), 165-191. (doi: 10.1007/s002050010127).

7 G. Bellettini, M. Novaga and M. Paolini. On a crystalline variational problem, part II: BVregularity and structure of minimizers on facets. Arch. Rational Mech. Anal. 157 (2001), 193-217. (doi: 10.1007/s002050100126).

8 G. Bellettini, G. Riey and M. Novaga. First variation of anisotropic energies and crystalline mean curvature for partitions. Interfaces Free Bound. 5 (2003), 331-356. (doi: 10.4171/IFB/82).

9 G. Bellettini. Anisotropic and Crystalline Mean Curvature Flow. In A Sampler of RiemannFinsler Geometry (eds. D. Bao, R. L. Bryant, S.-S. Chern, Z. Shen), Mathematical Sciences Research Institute Publications, vol. 50, pp. 49-83 (Cambridge Univ. Press, 2004).

10 G. Bellettini, V. Caselles, A. Chambolle and M. Novaga. Crystalline mean curvature flow of convex sets. Arch. Rational Mech. Anal. 179 (2005), 109-152. (doi: 10.1007/s00205-0050387-0). 
11 G. Bellettini, M. Chermisi and M. Novaga. Crystalline curvature flow of planar networks. Interfaces Free Bound. 8 (2006), 481-521. (doi: 10.4171/IFB/152).

12 G. Bellettini. Lecture notes on mean curvature flow, barriers and singular perturbations. vol. 12, (Pisa: Publications of the Scuola Normale Superiore di Pisa, 2013).

13 G. Bellettini, S. Y. Kholmatov. Minimizing movements for mean curvature flow of droplets with prescribed contact angle. J. Math. Pures Appl. 117 (2018), 1-58. (doi: 10.1016/j.matpur.2018.06.003).

14 G. Bellettini, S. Y. Kholmatov. Minimizing movements for mean curvature flow of partitions. SIAM J. Math. Anal. 50 (2018), 4117-4148. (doi: 10.1137/17M1159294).

15 K. Brakke. The motion of a surface by its mean curvature Math. Notes. vol. 20, (Princeton: Princeton University Press, 1978).

16 A. Chambolle. An algorithm for mean curvature motion. Interfaces Free Bound. 6 (2004), 195-218. (doi: 10.4171/IFB/97).

17 A. Chambolle, M. Morini, M. Novaga and M. Ponsiglione. Existence and uniqueness for anisotropic and crystalline mean curvature flows. J. Amer. Math. Soc. 32 (2019), 779-824. (doi: 10.1090/jams/919).

18 A. Chambolle, M. Morini and M. Ponsiglione. Nonlocal curvature flows. Arch. Rational Mech. Anal. 218 (2015), 1263-1329. (doi: 10.1007/s00205-015-0880-z).

19 A. Chambolle, M. Morini and M. Ponsiglione. Existence and uniqueness for a crystalline mean curvature flow. Comm. Pure Appl. Math. 70 (2017), 1084-1114. (doi: 10.1002/cpa.21668).

20 A. Chambolle, M. Morini, M. Novaga and M. Ponsiglione. Generalized crystalline evolutions as limits of flows with smooth anisotropies. Analysis PDE 12 (2019), 789-813. (doi: 10.2140/apde.2019.12.789).

21 E. De Giorgi. New problems on minimizing movements. Boundary value problems for PDEs and applications. RMA Res. Notes Appl. Math. 29 (1993), 81-98, Masson, Paris.

22 E. De Giorgi. Movimenti di partizioni. In Variational methods for free discontinuity structures, (eds. R. Serapioni, F. Tomarelli), vol. 25, pp. 1-5. (Basel: Birkhäuser, 1996). (doi: 10.1007/978-3-0348-9244-5_1).

23 D. Depner, H. Garcke and Y. Kohsaka. Mean curvature flow with triple junctions in higher space dimensions. Arch. Rational Mech. Anal. 211 (2014), 301-334. (doi: 10.1007/s00205013-0668-y).

24 L. Evans, H. Soner and P. Souganidis. Phase transitions and generalized motion by mean curvature. Comm. Pure Appl. Math. 45 (1992), 1097-1123. (doi: 10.1002/cpa.3160450903).

25 A. Freire. Mean curvature motion of graphs with constant contact angle at a free boundary. Anal. PDE 3 (2010), 359-407. (doi: 10.2140/apde.2010.3.359).

26 A. Freire. Mean curvature motion of triple junctions of graphs in two dimensions. Commun. Partial Differ. Equ. 35 (2010), 302-327. (doi: 10.1080/03605300903419775).

27 Y. Giga and M. E. Gurtin. A comparison theorem for crystalline evolutions in the plane. Quart. Appl. Math. 54 (1996), 727-737. (MathSciNet: MR1417236).

28 M.-H. Giga and Y. Giga. Crystalline and level set flow - convergence of a crystalline algorithm for a general anisotropic curvature flow in the plane. In Proc. Free Boundary Problems, Theory and Applications, (ed. N. Kenmochi), Gakuto International Series, Math. Sci. Appl., vol. 13, pp. 64-79 (Japan: Chiba, 2000).

29 Y. Giga, M. Paolini and P. Rybka. On the motion by singular interfacial energy. Japan J. Indust. Appl. Math. 18 (2001), 231-248. (doi:10.1007/BF03168572).

30 Y. Giga. Surface evolution equations (Basel: Birkhäuser, 2006).

31 Y. Giga and N. Požár. A level set crystalline mean curvature flow of surfaces. Adv. Differ. Equ. 21 (2016), 631-698. (MathSciNet: MR3493931).

32 Y. Giga and N. Požár. Approximation of general facets by regular facets with respect to anisotropic total variation energies and its application to crystalline mean curvature flow. Comm. Pure Appl. Math. 71 (2018), 1461-1491. (doi: 10.1002/cpa.21752).

33 M. A. Grayson. A short note on the evolution of a surface by its mean curvature. Duke Math. J. 58 (1989), 555-558. (doi: 10.1215/S0012-7094-89-05825-0).

34 G. Huisken. Asymptotic behaviour for singularities of the mean curvature flow. J. Differ. Geom. 31 (1990), 285-299. (doi: 10.4310/jdg/1214444099). 
35 G. Huisken. Local and global behaviour of hypersurfaces moving by mean curvature. Proc. Sympos. Pure Math. 54 (1993), 175-191. (MathSciNet: MR1216584).

36 G. Huisken. A distance comparison principle for evolving curves. Asian J. Math. 2 (1998), 127-133. (doi: 10.4310/AJM.1998.v2.n1.a2).

37 T. Ilmanen. Elliptic regularization and partial regularity for motion by mean curvature Mem. Amer. Math. Soc. 108 (1994) AMS.

38 T. Ilmanen, A. Neves and F. Schulze. On short time existence for the planar network flow. J. Differ. Geom. 111 (2019), 39-89. (doi:10.4310/jdg/1547607687).

39 L. Kim and Y. Tonegawa. On the mean curvature flow of grain boundaries. Ann. Inst. Fourier (Grenoble) 67 (2017), 43-142. (doi: 10.5802/aif.3077).

40 D. Kinderlehrer and C. Liu. Evolution of grain boundaries. Math. Models Methods Appl. Sci. 11 (2001), 713-729. (doi: 10.1142/S0218202501001069).

41 T. Laux and F. Otto. Convergence of the thresholding scheme for multi-phase mean curvature flow. Calc. Var. Partial Differ. Equ. 55 (2016), 55-129. (doi: 10.1007/s00526016-1053-0).

42 S. Luckhaus and T. Sturzenhecker. Implicit time discretization for the mean curvature flow equation. Calc. Var. Partial Differ. Equ. 3 (1995), 253-271. (doi: 10.1007/BF01205007).

43 F. Maggi. Sets of finite perimeter and geometric variational problems. An introduction to geometric measure theory (Cambridge: Cambridge University Press, 2012).

44 C. Mantegazza. Lecture notes on mean curvature flow. (Basel: Birkhäuser, 2011).

45 C. Mantegazza, M. Novaga, A. Pluda and F. Schulze. Evolution of networks with multiple junctions. arXiv:1611.08254 [math.DG].

46 B. Merriman, J. Bence and S. Osher. Diffusion generated motion by mean curvature (Los Angeles, CA: Manuscript, Department of Mathematics, University of California 1992).

47 L. Mugnai and M. Röger. The Allen-Cahn action functional in higher dimensions. Indiana Univ. Math. J. 10 (2008), 45-78. (doi: 10.4171/IFB/179).

48 L. Mugnai, C. Seis and E. Spadaro. Global solutions to the volume-preserving meancurvature flow. Calc. Var. 55 (2016), Article number 18. (doi: /10.1007/s00526-015-0943-x).

49 W. Mullins. Two-dimensional motion of idealized grain boundaries. J. Appl. Phys. 27 (1956), 900-904. (doi: 10.1007/978-3-642-59938-5_3).

50 F. Schulze and B. White. A local regularity theorem for mean curvature flow with triple edges. J. Reine Angew. Math. 758 (2020), 281-305. (doi: 10.1515/crelle-2017-0044).

51 J. E. Taylor, J. W. Cahn and C. A. Handwerker. Overview No. 98 I - Geometric models of crystal growth. Acta Metall. Mater. 40 (1992), 1443-1474. (doi: 10.1016/09567151(92)90090-2).

52 J. E. Taylor. Motion of curves by crystalline curvature, including triple junctions and boundary points. Differ Geom., Proc. Sympos. Pure Math. 54 (1993), 417-438. (doi: 10.1023/A:1004523005442).

53 J. E. Taylor. A variational approach to crystalline triple-junction motion. J. Statist. Phys. 95 (1999), 1221-1244. (doi: 10.1023/A:1004523005442).

54 Y. Tonegawa. Brakke's mean curvature flow. Springer briefs in bathematics (Singapore: Springer, 2019). 